\title{
Free vibration analysis for layered shells accounting of variable kinematic and thermo-mechanical coupling
}

\author{
S. Brischetto* and E. Carrera \\ Department of Aeronautics and Space Engineering, Politecnico di Torino, Italy
}

Received 21 April 2010

Revised 19 October 2010

\begin{abstract}
The free vibration analysis of one-layered and two-layered metallic cylindrical shell panels is evaluated in this work. The free frequency values are investigated for both thermo-mechanical and pure mechanical problems. Thermo-mechanical frequencies are calculated by means of a fully coupled thermo-mechanical model where both the displacement and temperature are primary variables in the considered governing equations. Pure mechanical frequencies are obtained from a mechanical model where the effect of the temperature field is not included in the stiffness matrix and the displacement is the only primary variable of the problem. The inclusion of the thermal part in the stiffness matrix gives larger frequencies. Both thermo-mechanical and pure mechanical models are developed in the framework of Carrera's Unified Formulation (CUF) in order to obtain several variable kinematic models. Both equivalent single layer and layer wise approaches are considered for multilayered shells. The use of refined two-dimensional theories for shells permits the evaluation of the effects of the thermo-mechanical coupling for lower and higher order modes, higher frequency values, multilayered configurations, thick and thin shells and several values of the radius of curvature of the shell geometry. It has mainly been concluded that the thermo-mechanical coupling is not influenced by the curvature of the shells, therefore, the main conclusions already given for the plate geometry are here confirmed: - the thermo-mechanical coupling is correctly determined if both the thermal and mechanical parts are correctly approximated; - it is small for each investigated case; - it influences the various vibration modes in different ways; - it has a limited dependence on the considered case, but this dependence vanishes if a global coupling is considered.
\end{abstract}

Keywords: Carrera's Unified Formulation, thermo-mechanical coupling, fully coupled thermo-mechanical models, refined theories, free vibrations of shells, global thermo-mechanical effect

\section{Introduction}

A fully coupled thermoelastic analysis permits to evaluate the effect of the temperature field on the deformation field and the changes in the temperature field due to the deformation of a body [1]: the thermo-mechanical coupling is not a one-way phenomenon. A fully coupled thermoelastic analysis considers both temperature and displacement fields as primary variables in the governing equations, as proposed in [2,3] for plate and shell geometries, respectively. The fully coupled thermo-mechanical governing equations directly give the displacements and the temperature through the thickness direction. In order to simplify the computational aspect of solving actual thermoelastic problems, it is possible to discount the coupling and to evaluate the temperature and deformation fields, in this order, separately. The thermoelastic problem, where the temperature and deformation fields are discounted, has been

\footnotetext{
*Corresponding author: Salvatore Brischetto, Department of Aeronautics and Space Engineering, Politecnico di Torino, Corso Duca degli Abruzzi, 24, 10129 Torino, Italy. Tel.: +39 011564 6869; Fax: +39 011564 6899; E-mail: salvatore.brischetto@polito.it.
} 
defined in $[2,3]$ as a partially coupled thermo-mechanical problem. Partially coupled thermo-mechanical models are extensively employed in the thermal stress analysis of aeronautical structures, such as one-layered and multilayered plates and shells, where the temperature variations are one of the most important factors, for the stress fields, that can cause failure of the structures [4]-[6]. However, partially coupled thermo-mechanical models cannot investigate the thermo-mechanical coupling effect in terms of frequencies, because they do not consider the thermal part in the stiffness matrix. Several partially coupled thermo-mechanical models have been developed for the static analysis of multilayered plates [7]-[9] and shells [10,11]. The thermal load is calculated imposing a priori linear temperature profile in the thickness direction or solving the Fourier's heat conduction equation.

In the present paper, the effect of thermo-mechanical coupling is investigated in the free vibration analysis of onelayered and two-layered metallic shells, in terms of frequency values, by using a fully coupled thermo-mechanical model. The use of refined two-dimensional theories for the free vibration analysis is mandatory for multilayered structures, moderately thick plates and shells, composite layers, higher order modes and higher frequency values (for details about the pure mechanical vibration analysis see [12], a preliminary discussion about the thermo-mechanical effects has been proposed in [13]). The effect of thermo-mechanical coupling in the free vibration analysis of one-layered and two-layered metallic plates in terms of frequency values has been investigated in [14]. In [14], a complete overview about the effects of the thickness ratio, layers stacking, higher order modes and high frequency values on the thermo-mechanical coupling is given. In the present paper, the same investigation is proposed for shell geometries in order to evaluate the effect of the curvature. Some results proposed in [3] give a preliminary study about the thermo-mechanical coupling for shells when the fundamental frequency is investigated for low values of wave number $(m=n=1$ ). Now the coupling is investigated for the higher frequencies related to the higher additional degrees of freedom of the considered refined model, and the the effect of the wave numbers is also accounted for. Moreover, a global energetic parameter is introduced in order to investigate the global thermo-mechanical effect.

\subsection{Literature review}

The fully-coupled thermo-mechanical analysis has been partially discussed in a small amount of work and only a few of these papers give numerical results about the static thermo-mechanical coupling. The physical behaviour of thermoelastic continuum is described in [15] by means of opportune variational principles: the stress equations of motion and the equation of heat conduction have been written as divergence equations, the strain-mechanical displacement relations and Fourier's heat conduction law have been written as gradient equations. This method has been extended to thermopiezoelectric mediums in [16] by simply adding the electrostatics charge equation in the divergence equations and the electric field-electric potential relations in the gradient equations. Das et alii [17] have obtained displacement and thermal fields in the Laplace transformation domain in order to avoid the use of the thermoelastic potential to solve the general problem of one-dimensional linearized simultaneous equations of thermoelasticity. Cannarozzi and Ubertini [18] have proposed a variational method for linear coupled quasi-static thermoelastic analysis, where the variational support is a statement in terms of displacement, temperature, stress and heat flux.

Papers concerning the dynamic thermo-mechanical analysis are not so numerous in the open literature, and no one gives a systematic numerical evaluation of the thermo-mechanical coupling in the free vibration analysis of multilayered shells. The present work gives a complete overview about the thermo-mechanical coupling in the free vibration analysis of shells. The previous authors' work [3] is mainly focused on the static analysis, and the free vibration problem is partially investigated by considering only the fundamental frequency: the investigation of higher order modes and higher frequency values introduces new effects completely different from those obtained when only the fundamental frequency is considered. Altay and Dökmeci [19] have given a three-dimensional theory of coupled thermoelasticity for a shell geometry. High-frequency vibrations of temperature-dependent materials have been investigated. The dynamic response of a thermally loaded elastic structure has been considered in [20]. The thermoelastic coupling term in a heat equation acts like a thermal source that is proportional to the strain rate. The thermoelastic coupling effect on the temperature and displacement fields has been measured as a function of the normalized frequency. Wilms and Cohen [21] have studied wave propagation in a thermoelastically coupled half space. Strain, temperature, stress and particle velocity have been obtained. Wauer [22] has investigated the dynamic interaction of magneto-thermo-elastic waves. The displacement and incremental temperature have been coupled 
and the thermo-elastic modes have been given. Considering a thermo-mechanical coupling, the obtained results demonstrate that, even though no mechanical damping has been considered, the eigenvalues have been characterized by their imaginary part (natural frequency) and also by their non vanishing (negative) real part (dissipation). A modified model of thermoelasticity, with an extra thermal stress effect and wave-type heat conduction, has been given by Kosinski and Frischmuth [23]. The model has been governed by a system of quasi-linear hyperbolic equations. All the essential material functions have been examined, and the impact of their nonlinearity on the solution of initial-boundary value problems has been studied. Thermoelastic vibrations of a free supported and clamped circular plate, caused by a thermal shock on the surface, have been analyzed in [24]. The partial differential equations of the coupled system have been reduced to Volterra's first and second kind of integral equations in the time domain. Very rapid thermal processes, under the action of a thermal shock, are interesting from the thermoelasticity point of view, since they require an analysis of the coupled temperature and deformation fields. This means that the temperature shock induces very rapid movements in the structure elements, thus causing the rise of very significant inertial forces, and, thereby, a rise in vibrations. Rapidly changeable contractions and expansions in oscillatory movements generate temperature changes in the material, which is susceptible to diffusion due to heat conduction. Yeh [25] has presented an analysis of large amplitude, thermo-mechanically coupled, vibrations of a simply supported orthotropic rectangular thin plate.

\subsection{Work plan}

The fully coupled thermo-mechanical governing equations permit the frequencies of the free vibration analysis of simply supported shells to be evaluated. Such frequencies are compared with the pure mechanical ones. The fully coupled governing equations are obtained by adding the internal thermal work to the Principle of Virtual Displacements (PVD). The employed refined two-dimensional theories are obtained as in Section 2; the displacement can be modelled in both Equivalent Single Layer (ESL) and Layer Wise (LW) form, but the temperature is always approximated in LW form. Section 3 gives the relations between the strain components and the displacement vector in the case of shell geometry, Section 4 illustrates the thermo-mechanical constitutive equations. PVD is extended to the fully coupled thermo-mechanical analysis in Section 5. The results for simply supported metallic one-layered and two-layered shells are discussed in Section 6. The main conclusions are given in Section 7.

\section{Carrera's Unified Formulation, CUF}

A large variety of shell theories are obtained, in a unified manner, by means of Carrera's Unified Formulation (CUF) [26,27]: the governing equations are written in terms of a few fundamental nuclei which do not formally depend on the order of expansion $N$ in the thickness direction and on the description of variables (equivalent single layer (ESL) or layer wise (LW)). A two-dimensional theory for shells permits the unknown variables to be expressed as a set of thickness functions that only depend on the thickness coordinate $z$ and the correspondent variable which depends on the in-plane coordinates $\alpha$ and $\beta$. The generic variable $f(\alpha, \beta, z)$, for instance the displacement or the temperature, and its variation $\delta \boldsymbol{f}(\alpha, \beta, z)$ are written according to the following general expansion:

$$
\begin{aligned}
& \boldsymbol{f}(\alpha, \beta, z)=F_{\tau}(z) \boldsymbol{f}_{\tau}(\alpha, \beta), \quad \delta \boldsymbol{f}(\alpha, \beta, z)=F_{s}(z) \delta \boldsymbol{f}_{s}(\alpha, \beta), \\
& \text { with } \tau, s=1, \ldots, N
\end{aligned}
$$

where the bold letters denote arrays, $(\alpha, \beta)$ are the in-plane curvilinear coordinates and $z$ the thickness one. The summing convention, with repeated indexes $\tau$ and $s$, is assumed. The order of expansion $N$ goes from first to fourth-order, and depending on the used thickness functions, a theory can be: ESL, when the variable is assumed for the whole multilayer and a Taylor expansion is employed as the thickness functions $F(z)$; LW, when the variable is considered independent in each layer and a combination of Legendre polynomials are used as the thickness functions $F(z)$. In the thermo-mechanical models, displacements can be modelled in both ESL or LW form, temperature is always considered in LW form. A two-dimensional thermo-mechanical model is defined therefore as ESL or LW, depending on the choice made for the displacement vector [13]. 


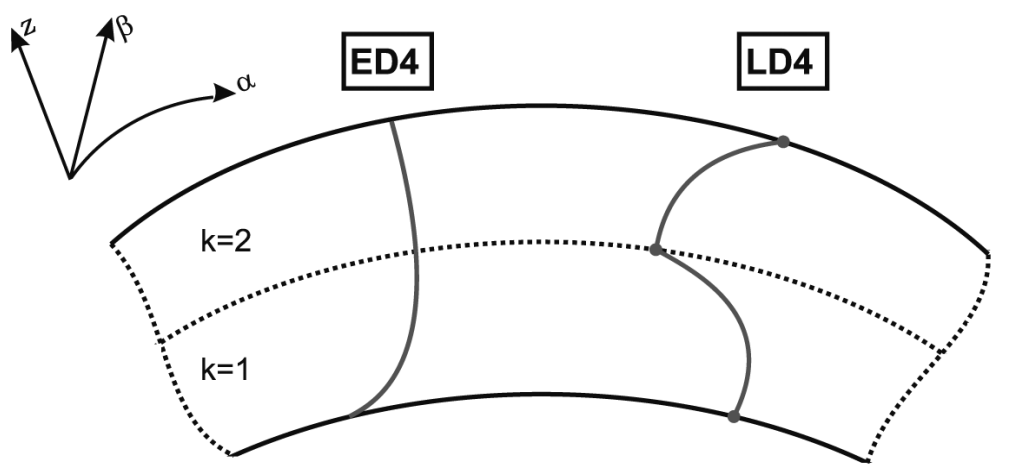

Fig. 1. Equivalent single layer and layer wise theories for a two-layered shell.

\subsection{Equivalent single layer approach}

The displacement $\boldsymbol{u}=(u, v, w)$ is described in equivalent single layer (ESL) form if the unknowns are the same for the whole multilayered shell [28] and they do not depend on the considered layer $k$ (see Fig. 1). The $z$ expansion is obtained via Taylor polynomials:

$$
\begin{aligned}
& u=F_{0} u_{0}+F_{1} u_{1}+\ldots+F_{N} u_{N}=F_{\tau} u_{\tau}, \\
& v=F_{0} v_{0}+F_{1} v_{1}+\ldots+F_{N} v_{N}=F_{\tau} v_{\tau}, \\
& w=F_{0} w_{0}+F_{1} w_{1}+\ldots+F_{N} w_{N}=F_{\tau} w_{\tau},
\end{aligned}
$$

with $\tau=0,1, \ldots, N ; N$ is the order of expansion that ranges from 1 (linear) to 4 :

$$
F_{0}=z^{0}=1, \quad F_{1}=z^{1}=z, \ldots, \quad F_{N}=z^{N} .
$$

Equation (2), written in a vectorial form, is:

$$
\begin{aligned}
& \boldsymbol{u}(\alpha, \beta, z)=F_{\tau}(z) \boldsymbol{u}_{\tau}(\alpha, \beta), \quad \delta \boldsymbol{u}(\alpha, \beta, z)=F_{s}(z) \delta \boldsymbol{u}_{s}(\alpha, \beta), \\
& \text { with } \tau, s=1, \ldots, N .
\end{aligned}
$$

Simpler theories, such those which discard the $\epsilon_{z z}$ effect, can be obtained from refined ESL models imposing that the transverse displacement $w$ is constant in $z$. First order Shear Deformation Theory (FSDT) [29] is obtained from an ESL model with linear expansion in the thickness direction $z$, by imposing a constant transverse displacement $w$ in $z$. Classical Lamination Theory (CLT) [30-32] is obtained from FSDT imposing an infinite transverse shear rigidity. All the ESL theories, with constant or linear transverse displacement $w$, which means zero or constant transverse normal strain $\epsilon_{z z}$, show Poisson's locking phenomena: it can be overcome via plane stress conditions in constitutive equations [33,34].

\subsection{Layer wise approach}

In a layer wise (LW) approach, each layer of a multilayered shell is described as independent shells [28]. The expansion of the displacement $\boldsymbol{u}^{k}=(u, v, w)^{k}$ is made for each layer $k$, and the zigzag form of displacement, in multilayered transverse-anisotropy shells, is easily obtained (see Fig. 1). The $z$ expansion for displacement components depends on each layer $k$ :

$$
\begin{aligned}
& u^{k}=F_{0} u_{0}^{k}+F_{1} u_{1}^{k}+\ldots+F_{N} u_{N}^{k}=F_{\tau} u_{\tau}^{k}, \\
& v^{k}=F_{0} v_{0}^{k}+F_{1} v_{1}^{k}+\ldots+F_{N} v_{N}^{k}=F_{\tau} v_{\tau}^{k}, \\
& w^{k}=F_{0} w_{0}^{k}+F_{1} w_{1}^{k}+\ldots+F_{N} w_{N}^{k}=F_{\tau} w_{\tau}^{k},
\end{aligned}
$$

with $\tau=0,1, \ldots, N, N$ is the order of expansion that ranges from 1 (linear) to $4 . k=1, \ldots, N_{l}$, where $N_{l}$ indicates the number of layers. The Eq. (5), in a vectorial form, is: 


$$
\begin{aligned}
& \boldsymbol{u}^{k}(\alpha, \beta, z)=F_{\tau}(z) \boldsymbol{u}_{\tau}^{k}(\alpha, \beta), \quad \delta \boldsymbol{u}^{k}(\alpha, \beta, z)=F_{s}(z) \delta \boldsymbol{u}_{s}^{k}(\alpha, \beta), \\
& \text { with } \tau, s=t, b, r \quad \text { and } k=1, \ldots, N_{l},
\end{aligned}
$$

where $t$ and $b$ indicate the top and bottom of each layer $k$, respectively; $r$ indicates the higher orders of expansion in the thickness direction: $r=2, \ldots, N$. The thickness functions $F_{\tau}\left(\zeta_{k}\right)$ and $F_{s}\left(\zeta_{k}\right)$ have now been defined at the $k$-layer level, they are a linear combination of Legendre polynomials $P_{j}=P_{j}\left(\zeta_{k}\right)$ of the $j^{t h}$-order defined in $\zeta_{k}$-domain $\left(\zeta_{k}=\frac{2 z_{k}}{h_{k}}\right.$ with $z_{k}$ local coordinate and $h_{k}$ thickness, both referred to $k^{\text {th }}$ layer, so $\left.-1 \leqslant \zeta_{k} \leqslant 1\right)$. The employed Legendre polynomials are:

$$
P_{0}=1, \quad P_{1}=\zeta_{k}, \quad P_{2}=\frac{\left(3 \zeta_{k}^{2}-1\right)}{2}, \quad P_{3}=\frac{5 \zeta_{k}{ }^{3}}{2}-\frac{3 \zeta_{k}}{2}, \quad P_{4}=\frac{35 \zeta_{k}{ }^{4}}{8}-\frac{15 \zeta_{k}{ }^{2}}{4}+\frac{3}{8},
$$

their combinations for the thickness functions are:

$$
F_{t}=F_{0}=\frac{P_{0}+P_{1}}{2}, \quad F_{b}=F_{1}=\frac{P_{0}-P_{1}}{2}, \quad F_{r}=P_{r}-P_{r-2} \quad \text { with } r=2, \ldots, N .
$$

The chosen functions have the following interesting properties:

$$
\begin{aligned}
& \zeta_{k}=1: F_{t}=1 ; \quad F_{b}=0 ; \quad F_{r}=0 \text { at top }, \\
& \zeta_{k}=-1: F_{t}=0 ; \quad F_{b}=1 ; \quad F_{r}=0 \text { at bottom, }
\end{aligned}
$$

that is interface values of the variables are considered as variable unknowns, see Fig. 1 . This feature permits to easily imposing the compatibility conditions for displacements at each layer interface. In LW models, when a multilayered anisotropic shell is considered, Poisson's locking phenomena does not appear even if a linear expansion in $z$ is considered for the transverse displacement $w$ : the transverse normal strain $\epsilon_{z z}$ is piece-wise constant in the thickness direction $[33,34]$.

In the case of thermo-mechanical problems, the primary variables are the displacement vector $\boldsymbol{u}=(u, v, w)$ and the scalar sovra-temperature $\theta$ (temperature $T_{1}$ referred to the reference external room temperature $T_{0}, \theta=T_{1}-T_{0}$ ). The variable $\theta^{k}$ is always modelled as LW because of the higher spatial gradient of the temperature field $[13,35]$ :

$$
\begin{aligned}
& \theta^{k}(\alpha, \beta, z)=F_{\tau}(z) \theta_{\tau}^{k}(\alpha, \beta), \quad \delta \theta^{k}(\alpha, \beta, z)=F_{s}(z) \delta \theta_{s}^{k}(\alpha, \beta), \\
& \text { with } \tau, s=t, b, r \quad \text { and } k=1, \ldots, N_{l},
\end{aligned}
$$

The thickness functions are a combination of Legendre polynomials as indicated in Eqs (7) and (8).

\section{Geometrical relations}

In [36] a thin shell is defined as a three-dimensional body bounded by two closely spaced curved surfaces, the distance between the two surfaces must be small in comparison with the other dimensions. The middle surface of the shell is the locus of points which lie midway between these surfaces. The distance between the surfaces measured along the normal to the middle surface is the thickness of the shell at that point. Details about the geometry and the reference system of a cylindrical shell panel are given in Fig. 2. The square of an infinitesimal linear segment in the layer, the associated infinitesimal area and volume are given by:

$$
\begin{aligned}
& d s_{k}^{2}=H_{\alpha}^{k^{2}} d \alpha_{k}^{2}+H_{\beta}^{k^{2}} d \beta_{k}^{2}+H_{z}^{k^{2}} d z_{k}^{2}, \\
& d \Omega_{k}=H_{\alpha}^{k} H_{\beta}^{k} d \alpha_{k} d \beta_{k}, \\
& d V_{k}=H_{\alpha}^{k} H_{\beta}^{k} H_{z}^{k} d \alpha_{k} d \beta_{k} d z_{k},
\end{aligned}
$$

where the metric coefficients, depending on the layer $k$ and on the thickness coordinate $z$, are:

$$
H_{\alpha}^{k}=A^{k}\left(1+z_{k} / R_{\alpha}^{k}\right), \quad H_{\beta}^{k}=B^{k}\left(1+z_{k} / R_{\beta}^{k}\right), \quad H_{z}^{k}=1 .
$$




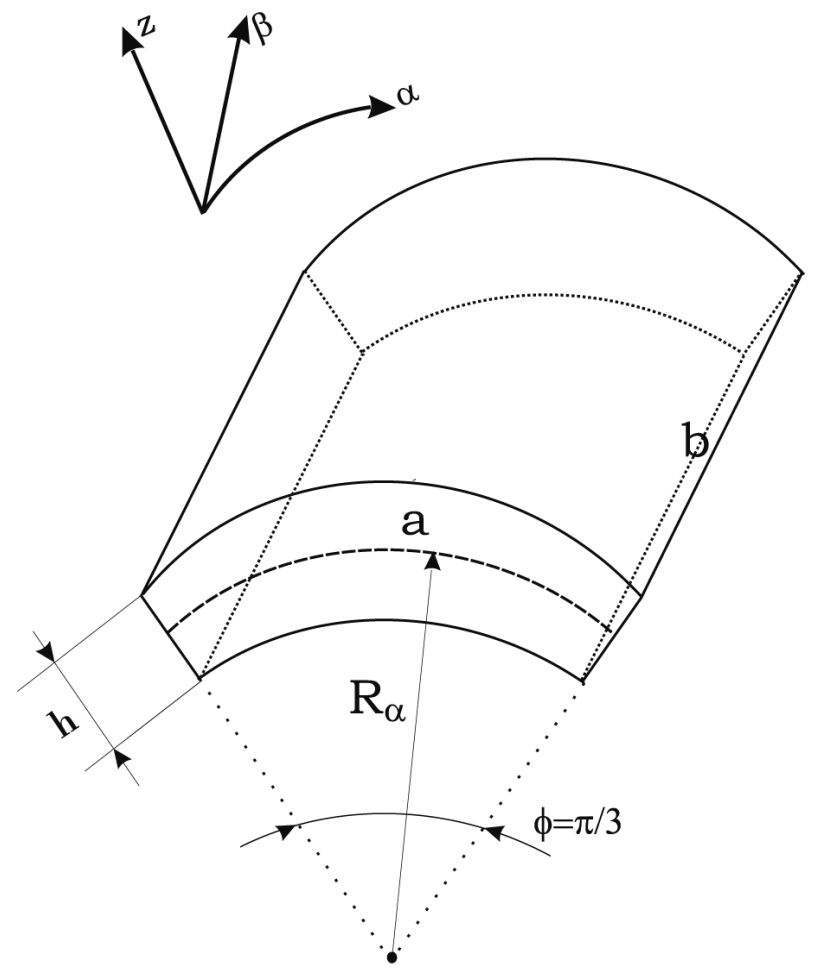

Fig. 2. Reference system and geometry for the considered shells.

$R_{\alpha}^{k}$ and $R_{\beta}^{k}$ are the principal radii of curvature along the coordinates $\alpha_{k}$ and $\beta_{k}$, respectively. $A^{k}$ and $B^{k}$ are the coefficients of the first fundamental form of $\Omega_{k}$ ( $\Gamma_{k}$ is the $\Omega_{k}$ boundary). If the attention is restricted to shells with constant radii of curvature (cylindrical, spherical, toroidal geometries), the coefficients $A^{k}$ and $B^{k}$ equal 1 . Further details for shells and their geometry are given in [36]. The geometrical relations for shells link the mechanical strains with the displacement vector. The relations split in in-plane $(p)$ and out-of-plane $(n)$ components are:

$$
\begin{aligned}
& \boldsymbol{\epsilon}_{p G}^{k}=\left[\epsilon_{\alpha \alpha}, \epsilon_{\beta \beta}, \gamma_{\alpha \beta}\right]^{k T}=\left(\boldsymbol{D}_{p}^{k}+\boldsymbol{A}_{p}^{k}\right) \boldsymbol{u}^{k}, \\
& \boldsymbol{\epsilon}_{n G}^{k}=\left[\gamma_{\alpha z}, \gamma_{\beta z}, \epsilon_{z z}\right]^{k T}=\left(\boldsymbol{D}_{n p}^{k}+\boldsymbol{D}_{n z}^{k}-\boldsymbol{A}_{n}^{k}\right) \boldsymbol{u}^{k},
\end{aligned}
$$

$\boldsymbol{\epsilon}_{p G}^{k}$ and $\boldsymbol{\epsilon}_{n G}^{k}$ are the in-plane and transverse strains, respectively. $\boldsymbol{u}^{k}=(u, v, w)^{k}$ is the displacement vector. $T$ means the transpose of a vector. The explicit form of the introduced arrays follows:

$$
\begin{aligned}
& \boldsymbol{D}_{p}^{k}=\left[\begin{array}{ccc}
\frac{\partial_{\alpha_{k}}}{H_{\alpha}^{k}} & 0 & 0 \\
0 & \frac{\partial_{\beta_{k}}}{H_{\beta}^{k}} & 0 \\
\frac{\partial_{\beta_{k}}}{H_{\beta}^{k}} & \frac{\partial_{\alpha_{k}}}{H_{\alpha}^{k}} & 0
\end{array}\right], \boldsymbol{D}_{n p}^{k}=\left[\begin{array}{ccc}
0 & 0 & \frac{\partial_{\alpha_{k}}}{H_{k}^{k}} \\
0 & 0 & \frac{\partial_{\beta_{k}}}{H_{\beta}^{k}} \\
0 & 0 & 0
\end{array}\right], \boldsymbol{D}_{n z}^{k}=\left[\begin{array}{ccc}
\partial_{z_{k}} & 0 & 0 \\
0 & \partial_{z_{k}} & 0 \\
0 & 0 & \partial_{z_{k}}
\end{array}\right], \\
& \boldsymbol{A}_{p}^{k}=\left[\begin{array}{lll}
0 & 0 & \frac{1}{H_{\alpha_{\alpha}}^{k} R_{\alpha}^{k}} \\
0 & 0 & \frac{1}{H_{\beta}^{k} R_{\beta}^{k}} \\
0 & 0 & 0
\end{array}\right], \boldsymbol{A}_{n}^{k}=\left[\begin{array}{ccc}
\frac{1}{H_{\alpha}^{k} R_{\alpha}^{k}} & 0 & 0 \\
0 & \frac{1}{H_{\beta}^{k} R_{\beta}^{k}} & 0 \\
0 & 0 & 0
\end{array}\right] .
\end{aligned}
$$

The symbols in differential operators matrices indicate the partial derivatives $\partial_{\alpha_{k}}=\frac{\partial}{\partial \alpha_{k}}, \partial_{\beta_{k}}=\frac{\partial}{\partial \beta_{k}}$ and $\partial_{z_{k}}=\frac{\partial}{\partial z_{k}}$. The parameters $H_{\alpha}^{k}$ and $H_{\beta}^{k}$ equal 1 in case of plates because the radii of curvature $R_{\alpha}^{k}$ and $R_{\beta}^{k}$ are infinite: matrices $\boldsymbol{A}_{p}^{k}$ and $\boldsymbol{A}_{n}^{k}$ are zero for plate geometries. 


\section{Constitutive equations}

Constitutive equations for the fully coupled thermo-mechanical problems have been obtained in [2] by using thermodynamical principles and Maxwell's relations [15,16,18,19]. Further details in the case of shell geometries can be found in [3]. A Gibbs free energy function and a thermomechanical enthalpy density [1,37] have been employed. Constitutive equations for the fully coupled thermomechanical vibrations are a particular case of the more general ones obtained in [2,3]:

$$
\begin{gathered}
\boldsymbol{\sigma}_{p C}^{k}=\boldsymbol{Q}_{p p}^{k} \boldsymbol{\epsilon}_{p G}^{k}+\boldsymbol{Q}_{p n}^{k} \boldsymbol{\epsilon}_{n G}^{k}-\boldsymbol{\lambda}_{p}^{k} \theta^{k} \\
\boldsymbol{\sigma}_{n C}^{k}=\boldsymbol{Q}_{n p}^{k} \boldsymbol{\epsilon}_{p G}^{k}+\boldsymbol{Q}_{n n}^{k} \boldsymbol{\epsilon}_{n G}^{k}-\boldsymbol{\lambda}_{n}^{k} \theta^{k} \\
\eta_{C}^{k}=\boldsymbol{\lambda}_{p}^{k T} \boldsymbol{\epsilon}_{p G}^{k}+\boldsymbol{\lambda}_{n}^{k T} \boldsymbol{\epsilon}_{n G}^{k}+\chi^{k} \theta^{k}
\end{gathered}
$$

with in-plane $(p)$ and out-of-plane $(n)$ stress and strain components for each layer $k$ :

$$
\boldsymbol{\sigma}_{p C}^{k}=\left\{\begin{array}{l}
\sigma_{\alpha \alpha} \\
\sigma_{\beta \beta} \\
\sigma_{\alpha \beta}
\end{array}\right\}^{k}, \quad \boldsymbol{\sigma}_{n C}^{k}=\left\{\begin{array}{l}
\sigma_{\alpha z} \\
\sigma_{\beta z} \\
\sigma_{z z}
\end{array}\right\}^{k}, \quad \boldsymbol{\epsilon}_{p G}^{k}=\left\{\begin{array}{l}
\epsilon_{\alpha \alpha} \\
\epsilon_{\beta \beta} \\
\gamma_{\alpha \beta}
\end{array}\right\}^{k}, \boldsymbol{\epsilon}_{n G}^{k}=\left\{\begin{array}{l}
\gamma_{\alpha z} \\
\gamma_{\beta z} \\
\epsilon_{z z}
\end{array}\right\}^{k} .
$$

The sovra-temperature $\theta^{k}$, the term $\chi^{k}=\frac{\rho^{k} C_{v}^{k}}{T_{0}}$ and the entropy for unite volume $\eta^{k}$ are scalar variables in each layer $k . \rho^{k}$ is the material density, $C_{v}^{k}$ is the specific heat per unit mass and $T_{0}$ is the reference temperature [2]. The explicit forms of the split matrices in Eqs (20)-(22) are:

- Elastic coefficients matrices:

$$
\begin{aligned}
& \boldsymbol{Q}_{p p}^{k}=\left[\begin{array}{lll}
Q_{11} & Q_{12} & Q_{16} \\
Q_{12} & Q_{22} & Q_{26} \\
Q_{16} & Q_{26} & Q_{66}
\end{array}\right]^{k}, \boldsymbol{Q}_{p n}^{k}=\left[\begin{array}{lll}
0 & 0 & Q_{13} \\
0 & 0 & Q_{23} \\
0 & 0 & Q_{36}
\end{array}\right]^{k}, \\
& \boldsymbol{Q}_{n p}^{k}=\left[\begin{array}{ccc}
0 & 0 & 0 \\
0 & 0 & 0 \\
Q_{13} & Q_{23} & Q_{36}
\end{array}\right]^{k}, \boldsymbol{Q}_{n n}^{k}=\left[\begin{array}{ccc}
Q_{55} & Q_{45} & 0 \\
Q_{45} & Q_{44} & 0 \\
0 & 0 & Q_{33}
\end{array}\right]^{k} .
\end{aligned}
$$

- Thermo-mechanical coupling coefficients:

$$
\boldsymbol{\lambda}_{p}^{k}=\boldsymbol{Q}_{p p}^{k} \boldsymbol{\alpha}_{p}^{k}+\boldsymbol{Q}_{p n}^{k} \boldsymbol{\alpha}_{n}^{k}=\left[\begin{array}{c}
\lambda_{1} \\
\lambda_{2} \\
\lambda_{6}
\end{array}\right]^{k}, \quad \boldsymbol{\lambda}_{n}^{k}=\boldsymbol{Q}_{n p}^{k} \boldsymbol{\alpha}_{p}^{k}+\boldsymbol{Q}_{n n}^{k} \boldsymbol{\alpha}_{n}^{k}=\left[\begin{array}{c}
0 \\
0 \\
\lambda_{3}
\end{array}\right]^{k}
$$

where $\boldsymbol{\alpha}_{p}^{k T}=\left(\alpha_{1}, \alpha_{2}, 0\right)$ and $\boldsymbol{\alpha}_{n}^{k T}=\left(0,0, \alpha_{3}\right)$ are the thermal expansion coefficients split in in-plane and outof-plane components. In Eqs (20)-(22) subscript $C$ means constitutive equations, $G$ means the use of geometrical relations for shells of Section 3.

\section{Governing equations}

The variational statement for the thermo-mechanical vibrations includes the internal thermal work $\left(\delta \theta^{k} \eta_{C}^{k}\right)$ in the classical principle of virtual displacements [2,3]:

$$
\sum_{k=1}^{N_{l}} \int_{\Omega_{k}} \int_{A_{k}}\left\{\delta \boldsymbol{\epsilon}_{p G}^{k}{ }^{T} \boldsymbol{\sigma}_{p C}^{k}+\delta \boldsymbol{\epsilon}_{n G}^{k}{ }^{T} \boldsymbol{\sigma}_{n C}^{k}-\delta \theta^{k} \eta_{C}^{k}\right\} d \Omega_{k} d z=-\sum_{k=1}^{N_{l}} \delta L_{i n}^{k},
$$

where $N_{l}$ are the layers of the considered multilayered shell, $\Omega_{k}$ is the in-plane surface domain and $A_{k}$ is the thickness direction domain. $\delta L_{i n}^{k}$ is the inertial virtual work at the $k$-layer level. 
By considering the constitutive equations as obtained in Eqs (20)-(22), the geometrical relations for shells as obtained in Section 3 and Carrera's Unified Formulation as described in Section 2, the Eq. (26) is rewritten in the following form for a generic layer $k$ :

$$
\begin{aligned}
& \int_{\Omega_{k}} \int_{A_{k}}\left[\left(\left(\boldsymbol{D}_{p}^{k}+\boldsymbol{A}_{p}^{k}\right) F_{s} \delta \boldsymbol{u}_{s}^{k}\right)^{T}\left(\left(\boldsymbol{Q}_{p p}^{k}\left(\boldsymbol{D}_{p}^{k}+\boldsymbol{A}_{p}^{k}\right)+\boldsymbol{Q}_{p n}^{k}\left(\boldsymbol{D}_{n p}^{k}+\boldsymbol{D}_{n z}^{k}-\boldsymbol{A}_{n}^{k}\right)\right) F_{\tau} \boldsymbol{u}_{\tau}^{k}-\boldsymbol{\lambda}_{p}^{k} F_{\tau} \theta_{\tau}^{k}\right)\right. \\
& +\left(\left(\boldsymbol{D}_{n p}^{k}+\boldsymbol{D}_{n z}^{k}-\boldsymbol{A}_{n}^{k}\right) F_{s} \delta \boldsymbol{u}_{s}^{k}\right)^{T}\left(\left(\boldsymbol{Q}_{n p}^{k}\left(\boldsymbol{D}_{p}^{k}+\boldsymbol{A}_{p}^{k}\right)+\boldsymbol{Q}_{n n}^{k}\left(\boldsymbol{D}_{n p}^{k}+\boldsymbol{D}_{n z}^{k}-\boldsymbol{A}_{n}^{k}\right)\right) F_{\tau} \boldsymbol{u}_{\tau}^{k}-\boldsymbol{\lambda}_{n}^{k} F_{\tau} \theta_{\tau}^{k}\right) \\
& \left.-F_{s} \delta \theta_{s}^{k T}\left(\left(\boldsymbol{\lambda}_{p}^{k T}\left(\boldsymbol{D}_{p}^{k}+\boldsymbol{A}_{p}^{k}\right)+\boldsymbol{\lambda}_{n}^{k T}\left(\boldsymbol{D}_{n p}^{k}+\boldsymbol{D}_{n z}^{k}-\boldsymbol{A}_{n}^{k}\right)\right) F_{\tau} \boldsymbol{u}_{\tau}^{k}+\chi^{k} F_{\tau} \theta_{\tau}^{k}\right)\right] d \Omega_{k} d z=-\delta L_{i n}^{k} .
\end{aligned}
$$

Integrating by parts the Eq. (27), as suggested in [7,11], the fundamental nuclei $\boldsymbol{K}_{u u}^{k \tau s}, \boldsymbol{K}_{u \theta}^{k \tau s}, \boldsymbol{K}_{\theta u}^{k \tau s}, \boldsymbol{K}_{\theta \theta}^{k \tau s}$, and that for the inertial matrix $\boldsymbol{M}_{u u}^{k \tau s}$ are obtained by using the governing equations in the following form:

$$
\begin{array}{ll}
\delta \boldsymbol{u}_{s}^{k}: & \boldsymbol{K}_{u u}^{k \tau s} \boldsymbol{u}_{\tau}^{k}+\boldsymbol{K}_{u \theta}^{k \tau s} \theta_{\tau}^{k}=-\boldsymbol{M}_{u u}^{k \tau s} \ddot{\boldsymbol{u}}_{\tau}^{k} \\
\delta \theta_{s}^{k}: & \boldsymbol{K}_{\theta u}^{k \tau s} \boldsymbol{u}_{\tau}^{k}+\boldsymbol{K}_{\theta \theta}^{k \tau s} \theta_{\tau}^{k}=0 .
\end{array}
$$

$\boldsymbol{u}_{\tau}^{k}$ is the vector for the degrees of freedom of the displacement, $\theta_{\tau}^{k}$ is the vector for the degrees of freedom of the sovra-temperature, and $\ddot{\boldsymbol{u}}_{\tau}^{k}$ is the second temporal derivative of $\boldsymbol{u}_{\tau}^{k}$. Along with these governing equations the following boundary conditions on the edge $\Gamma_{k}$ of the in-plane integration domain $\Omega_{k}$ hold:

$$
\begin{aligned}
& \Pi_{u u}^{k \tau s} \boldsymbol{u}_{\tau}^{k}+\Pi_{u \theta}^{k \tau s} \theta_{\tau}^{k}=\Pi_{u u}^{k \tau s} \overline{\boldsymbol{u}}_{\tau}^{k}+\Pi_{u \theta}^{k \tau s} \bar{\theta}_{\tau}^{k} \\
& \Pi_{\theta u}^{k \tau s} \boldsymbol{u}_{\tau}^{k}+\Pi_{\theta \theta}^{k \tau s} \theta_{\tau}^{k}=\Pi_{\theta u}^{k \tau s} \overline{\boldsymbol{u}}_{\tau}^{k}+\Pi_{\theta \theta}^{k \tau s} \bar{\theta}_{\tau}^{k} .
\end{aligned}
$$

As indicated in [2,3], the sovra-temperature $\theta^{k}$ is a variable of the problem (always in LW form). The displacements $\boldsymbol{u}^{k}$ can be seen in ESL or LW form.

Governing equations for the pure mechanical problem are obtained from Eq. (28) simply discarding the thermal part:

$$
\delta \boldsymbol{u}_{s}^{k}: \quad \boldsymbol{K}_{u u}^{k \tau s} \boldsymbol{u}_{\tau}^{k}=-\boldsymbol{M}_{u u}^{k \tau s} \ddot{\boldsymbol{u}}_{\tau}^{k}
$$

with

$$
\Pi_{u u}^{k \tau s} \boldsymbol{u}_{\tau}^{k}=\Pi_{u u}^{k \tau s} \overline{\boldsymbol{u}}_{\tau}^{k} .
$$

Fundamental nuclei for the thermo-mechanical vibration problem are:

$$
\begin{aligned}
\boldsymbol{K}_{u u}^{k \tau s}= & \int_{A_{k}}\left[\left(-\boldsymbol{D}_{p}^{k}+\boldsymbol{A}_{p}^{k}\right)^{T}\left(\boldsymbol{Q}_{p p}^{k}\left(\boldsymbol{D}_{p}^{k}+\boldsymbol{A}_{p}^{k}\right)+\boldsymbol{Q}_{p n}^{k}\left(\boldsymbol{D}_{n p}^{k}+\boldsymbol{D}_{n z}^{k}-\boldsymbol{A}_{n}^{k}\right)\right)+\right. \\
& \left.\left(-\boldsymbol{D}_{n p}^{k}+\boldsymbol{D}_{n z}^{k}-\boldsymbol{A}_{n}^{k}\right)^{T}\left(\boldsymbol{Q}_{n p}^{k}\left(\boldsymbol{D}_{p}^{k}+\boldsymbol{A}_{p}^{k}\right)+\boldsymbol{Q}_{n n}^{k}\left(\boldsymbol{D}_{n p}^{k}+\boldsymbol{D}_{n z}^{k}-\boldsymbol{A}_{n}^{k}\right)\right)\right] F_{s} F_{\tau} H_{\alpha}^{k} H_{\beta}^{k} d z, \\
\boldsymbol{K}_{u \theta}^{k \tau s}= & \int_{A_{k}}\left[\left(-\boldsymbol{D}_{p}^{k}+\boldsymbol{A}_{p}^{k}\right)^{T}\left(-\boldsymbol{\lambda}_{p}^{k}\right)+\left(-\boldsymbol{D}_{n p}^{k}+\boldsymbol{D}_{n z}^{k}-\boldsymbol{A}_{n}^{k}\right)^{T}\left(-\boldsymbol{\lambda}_{n}^{k}\right)\right] F_{s} F_{\tau} H_{\alpha}^{k} H_{\beta}^{k} d z, \\
\boldsymbol{K}_{\theta u}^{k \tau s}= & \int_{A_{k}}\left[-\boldsymbol{\lambda}_{p}^{k T}\left(\boldsymbol{D}_{p}^{k}+\boldsymbol{A}_{p}^{k}\right)-\boldsymbol{\lambda}_{n}^{k T}\left(\boldsymbol{D}_{n p}^{k}+\boldsymbol{D}_{n z}^{k}-\boldsymbol{A}_{n}^{k}\right)\right] F_{s} F_{\tau} H_{\alpha}^{k} H_{\beta}^{k} d z, \\
\boldsymbol{K}_{\theta \theta}^{k \tau s}= & \int_{A_{k}}\left[-\chi^{k}\right] F_{s} F_{\tau} H_{\alpha}^{k} H_{\beta}^{k} d z, \\
\boldsymbol{M}_{u u}^{k \tau s}= & \int_{A_{k}}\left(\rho^{k} \boldsymbol{I}\right) F_{s} F_{\tau} H_{\alpha}^{k} H_{\beta}^{k} d z .
\end{aligned}
$$


$\boldsymbol{I}$ is the $(3 \times 3)$ identity matrix. Nuclei for boundary conditions on the edge $\Gamma_{k}$ state:

$$
\begin{aligned}
\Pi_{u u}^{k \tau s}= & \int_{A_{k}}\left[\boldsymbol{I}_{p}^{k T}\left(\boldsymbol{Q}_{p p}^{k}\left(\boldsymbol{D}_{p}^{k}+\boldsymbol{A}_{p}^{k}\right)+\boldsymbol{Q}_{p n}^{k}\left(\boldsymbol{D}_{n p}^{k}+\boldsymbol{D}_{n z}^{k}-\boldsymbol{A}_{n}^{k}\right)\right)+\boldsymbol{I}_{n p}^{k T}\left(\boldsymbol{Q}_{n p}^{k}\left(\boldsymbol{D}_{p}^{k}+\boldsymbol{A}_{p}^{k}\right)\right.\right. \\
& \left.\left.+\boldsymbol{Q}_{n n}^{k}\left(\boldsymbol{D}_{n p}^{k}+\boldsymbol{D}_{n z}^{k}-\boldsymbol{A}_{n}^{k}\right)\right)\right] F_{s} F_{\tau} H_{\alpha}^{k} H_{\beta}^{k} d z \\
\Pi_{u \theta}^{k \tau s}= & \int_{A_{k}}\left[\boldsymbol{I}_{p}^{k T}\left(-\boldsymbol{\lambda}_{p}^{k}\right)+\boldsymbol{I}_{n p}^{k T}\left(-\boldsymbol{\lambda}_{n}^{k}\right)\right] F_{s} F_{\tau} H_{\alpha}^{k} H_{\beta}^{k} d z \\
\Pi_{\theta u}^{k \tau s}= & \Pi_{\theta \theta}^{k \tau s}=0 .
\end{aligned}
$$

Matrices $\boldsymbol{I}_{p}^{k}$ and $\boldsymbol{I}_{n p}^{k}$ are obtained from matrices $\boldsymbol{D}_{p}^{k}$ and $\boldsymbol{D}_{n p}^{k}$ simply replacing the differential operators with 1. In the case of pure mechanical vibrations, only nuclei $\boldsymbol{K}_{u u}^{k \tau s}, \boldsymbol{M}_{u u}^{k \tau s}$ and $\Pi_{u u}^{k \tau s}$ in Eqs (31), (35) and (36), respectively, are considered.

In order to write the explicit form of fundamental nuclei obtained in this section, the following integrals in the $z$-thickness direction must be defined:

$$
\begin{aligned}
& \left(J^{k \tau s}, J_{\alpha}^{k \tau s}, J_{\beta}^{k \tau s}, J_{\frac{\alpha}{\beta}}^{k \tau s}, J_{\frac{\beta}{\alpha}}^{k \tau s}, J_{\alpha \beta}^{k \tau s}\right)=\int_{A_{k}} F_{\tau} F_{s}\left(1, H_{\alpha}^{k}, H_{\beta}^{k}, \frac{H_{\alpha}^{k}}{H_{\beta}^{k}}, \frac{H_{\beta}^{k}}{H_{\alpha}^{k}}, H_{\alpha}^{k} H_{\beta}^{k}\right) d z \\
& \left(J^{k \tau_{z} s}, J_{\alpha}^{k \tau_{z} s}, J_{\beta}^{k \tau_{z} s}, J_{\frac{\alpha}{\beta}}^{k \tau_{z} s}, J_{\frac{\beta}{\alpha}}^{k \tau_{z} s}, J_{\alpha \beta}^{k \tau_{z} s}\right)=\int_{A_{k}} \frac{\partial F_{\tau}}{\partial z} F_{s}\left(1, H_{\alpha}^{k}, H_{\beta}^{k}, \frac{H_{\alpha}^{k}}{H_{\beta}^{k}}, \frac{H_{\beta}^{k}}{H_{\alpha}^{k}}, H_{\alpha}^{k} H_{\beta}^{k}\right) d z, \\
& \left(J^{k \tau s_{z}}, J_{\alpha}^{k \tau s_{z}}, J_{\beta}^{k \tau s_{z}}, J_{\frac{\alpha}{\beta}}^{k \tau s_{z}}, J_{\frac{\beta}{\alpha}}^{k \tau s_{z}}, J_{\alpha \beta}^{k \tau s_{z}}\right)=\int_{A_{k}} F_{\tau} \frac{\partial F_{s}}{\partial z}\left(1, H_{\alpha}^{k}, H_{\beta}^{k}, \frac{H_{\alpha}^{k}}{H_{\beta}^{k}}, \frac{H_{\beta}^{k}}{H_{\alpha}^{k}}, H_{\alpha}^{k} H_{\beta}^{k}\right) d z \\
& \left(J^{k \tau_{z} s_{z}}, J_{\alpha}^{k \tau_{z} s_{z}}, J_{\beta}^{k \tau_{z} s_{z}}, J_{\frac{\alpha}{\beta}}^{k \tau_{z} s_{z}}, J_{\frac{\beta}{\alpha}}^{k \tau_{z} s_{z}}, J_{\alpha \beta}^{k \tau_{z} s_{z}}\right)=\int_{A_{k}} \frac{\partial F_{\tau}}{\partial z} \frac{\partial F_{s}}{\partial z}\left(1, H_{\alpha}^{k}, H_{\beta}^{k}, \frac{H_{\alpha}^{k}}{H_{\beta}^{k}}, \frac{H_{\beta}^{k}}{H_{\alpha}^{k}}, H_{\alpha}^{k} H_{\beta}^{k}\right) d z .
\end{aligned}
$$

By using the Eqs (39) and developing the matrices products, the explicit forms of fundamental nuclei are obtained.

Navier-type closed form solution is obtained via substitution of harmonic expressions for the displacements and temperature as well as considering the following material coefficients equal zero: $Q_{16}=Q_{26}=Q_{36}=Q_{45}=$ 0 and $\lambda_{6}=0$. The following harmonic assumptions can be made for the variables, which correspond to simply supported boundary conditions:

$$
\begin{aligned}
& u_{\tau}^{k}=\sum_{m, n}\left(\hat{U}_{\tau}^{k}\right) \cos \left(\frac{m \pi \alpha_{k}}{a_{k}}\right) \sin \left(\frac{n \pi \beta_{k}}{b_{k}}\right), \quad k=1, N_{l}, \\
& v_{\tau}^{k}=\sum_{m, n}\left(\hat{V}_{\tau}^{k}\right) \sin \left(\frac{m \pi \alpha_{k}}{a_{k}}\right) \cos \left(\frac{n \pi \beta_{k}}{b_{k}}\right), \quad \tau=t, b, r, \\
& \left(w_{\tau}^{k}, \theta_{\tau}^{k}\right)=\sum_{m, n}\left(\hat{W}_{\tau}^{k}, \hat{\theta}_{\tau}^{k}\right) \sin \left(\frac{m \pi \alpha_{k}}{a_{k}}\right) \sin \left(\frac{n \pi \beta_{k}}{b_{k}}\right), \quad r=2, N,
\end{aligned}
$$

where $\hat{U}_{\tau}^{k}, \hat{V}_{\tau}^{k}, \hat{W}_{\tau}^{k}, \hat{\theta}_{\tau}^{k}$ are the amplitudes, $m$ and $n$ are the wave numbers in $\alpha$ and $\beta$ directions, respectively, $a$ and $b$ are the shell dimensions, $k$ indicates the considered layer. The explicit algebraic closed-form of nuclei in Eqs (31)-(35) is not here given for sake of brevity, but it can be found in [3]. 
Table 1

Number of frequencies (depending on $N D O F$ in the thickness direction) for fixed wave numbers $(m, n)$ in the in-plane directions. $N D O F=\left(\left(N \times N_{l}\right)+1\right) \times 3$ in the case of $\mathrm{LW}$ theories, where $N_{l}$ is the number of layers

\begin{tabular}{lcc}
\hline Theory & \multicolumn{2}{c}{ NDOF } \\
\cline { 2 - 3 } & $N_{l}=1$ & $N_{l}=2$ \\
\hline CLT & 3 & 3 \\
FSDT & 5 & 5 \\
ESL $(N=4)$ & 15 & 15 \\
LW $(N=2)$ & 9 & 15 \\
LW $(N=4)$ & 15 & 27 \\
\hline
\end{tabular}

Table 2

Free vibrations of one-layered isotropic shell: fundamental frequency $f$ in $\mathrm{Hz}$ for $m=$ $n=1$. Thermo-mechanical coupling parameter $\Delta(\%)=\frac{f_{T M}-f}{f} \times 100$ is put in brackets

\begin{tabular}{lcccc}
\hline$R_{\alpha} / h$ & 5 & 10 & 50 & 100 \\
\hline LD4 & 1413.5 & 1235.3 & 465.79 & 253.64 \\
LD4(TM) & $1415.3(0.127)$ & $1238.0(0.219)$ & $467.78(0.427)$ & $254.75(0.438)$ \\
LD2 & 1483.5 & 1281.9 & 468.42 & 254.01 \\
LD2(TM) & $1484.8(0.088)$ & $1284.5(0.203)$ & $470.43(0.429)$ & $255.13(0.441)$ \\
FSDT & 1483.1 & 1281.0 & 468.38 & 254.02 \\
FSDT(TM) & $1485.6(0.169)$ & $1286.0(0.390)$ & $472.42(0.862)$ & $256.27(0.886)$ \\
CLT & 1590.4 & 1590.4 & 488.19 & 256.67 \\
CLT(TM) & $1590.4(0.000)$ & $1590.4(0.000)$ & $492.78(0.940)$ & $259.00(0.908)$ \\
\hline
\end{tabular}

Table 3

Free vibrations of one-layered isotropic shell: fundamental frequency $f$ in $H z$ for $m=n=$ 10. Thermo-mechanical coupling parameter $\Delta(\%)=\frac{f_{T M}-f}{f} \times 100$ is put in brackets

\begin{tabular}{lcccc}
\hline$R_{\alpha} / h$ & 5 & 10 & 50 & 100 \\
\hline LD4 & 15808 & 15592 & 14124 & 12337 \\
LD4(TM) & $15810(0.013)$ & $15598(0.038)$ & $14142(0.127)$ & $12363(0.211)$ \\
LD2 & 15889 & 15854 & 14829 & 12805 \\
LD2(TM) & $15889(0.000)$ & $15855(0.006)$ & $14842(0.088)$ & $12830(0.195)$ \\
S FSDT & 15891 & 15854 & 14822 & 12795 \\
FSDT(TM) & $15891(0.000)$ & $15854(0.006)$ & $14847(0.169)$ & $12845(0.391)$ \\
CLT & 15904 & 15905 & 15905 & 15905 \\
CLT(TM) & $15904(0.000)$ & $15905(0.000)$ & $15905(0.000)$ & $15905(0.000)$ \\
\hline
\end{tabular}

\subsection{Acronyms}

The refined two-dimensional models developed for the thermo-mechanical analysis of multilayered shells are defined by a system of acronyms. A two-dimensional model is defined as ESL or LW depending on the choice made for the displacement because the temperature is always considered in LW form. ESL models are indicated as ED1-ED4, where E means the ESL approach, D means that the extension of the Principle of Virtual Displacements to thermo-mechanical analysis has been employed; the last digit, from 1 to 4 , indicates the order of expansion in the thickness direction for both displacements and temperature. In the case of LW models, the letter $\mathrm{E}$ is replaced by a letter L, therefore the relative models are indicated as LD1-LD4. In the case of a thermo-mechanical problem an additional parenthesis is introduced in the acronyms to indicate the fully coupled thermo(T)-mechanical(M) analysis. No parenthesis are added in the case of a pure mechanical problem. CLT and FSDT have been proposed as classical theories in order to evaluate the thickness stretching effect in the performed analysis.

\section{Results}

The results given in this section, consider a cylindrical shell panel which is simply supported (see Fig. 2). The radius of curvature in $\beta$ direction is $R_{\beta}=\infty$, the radius of curvature in $\alpha$ direction is $R_{\alpha}=10 \mathrm{~m}$ with angle $\Phi$ equals $\frac{\pi}{3}$. The in-plane shell dimensions are $a=\frac{\pi}{3} R_{\alpha}=10.47197551 \mathrm{~m}$ and $b=1 \mathrm{~m}$. The investigated thickness ratios 
Table 4

Free vibrations of one-layered isotropic shell: fundamental frequency $f$ in $H z$ for $m=n=$ 50. Thermo-mechanical coupling parameter $\Delta(\%)=\frac{f_{T M}-f}{f} \times 100$ is put in brackets

\begin{tabular}{lcccc}
\hline$R_{\alpha} / h$ & 5 & 10 & 50 & 100 \\
\hline LD4 & 79459 & 79430 & 77974 & 75485 \\
LD4(TM) & $79460(0.001)$ & $79432(0.002)$ & $78003(0.037)$ & $75546(0.081)$ \\
LD2 & 79478 & 79502 & 79272 & 78536 \\
LD2(TM) & $79478(0.000)$ & $79503(0.001)$ & $79276(0.005)$ & $78550(0.018)$ \\
FSDT & 79490 & 79510 & 79270 & 78526 \\
FSDT(TM) & $79490(0.000)$ & $79510(0.000)$ & $79277(0.009)$ & $78554(0.036)$ \\
CLT & 79522 & 79525 & 79526 & 79526 \\
CLT(TM) & $79522(0.000)$ & $79525(0.000)$ & $79526(0.000)$ & $79526(0.000)$ \\
\hline
\end{tabular}

Table 5

Free vibrations of two-layered isotropic shell: fundamental frequency $f$ in $H z$ for $m=$ $n=1$. Thermo-mechanical coupling parameter $\Delta(\%)=\frac{f_{T M}-f}{f} \times 100$ is put in brackets

\begin{tabular}{lcccc}
\hline$R_{\alpha} / h$ & 5 & 10 & 50 & 100 \\
\hline LD4 & 1371.8 & 1201.1 & 454.22 & 247.89 \\
LD4(TM) & $1372.5(0.051)$ & $1202.4(0.108)$ & $455.28(0.233)$ & $248.41(0.210)$ \\
ED4 & 1378.2 & 1205.0 & 455.44 & 247.93 \\
ED4(TM) & $1378.8(0.043)$ & $1206.3(0.108)$ & $455.50(0.233)$ & $248.44(0.206)$ \\
FSDT & 1453.0 & 1254.0 & 457.10 & 248.30 \\
FSDT(TM) & $1454.4(0.096)$ & $1257.0(0.239)$ & $459.28(0.477)$ & $249.35(0.423)$ \\
CLT & 1557.6 & 1559.2 & 476.14 & 250.84 \\
CLT(TM) & $1557.6(0.000)$ & $1559.3(0.006)$ & $478.65(0.527)$ & $251.94(0.438)$ \\
\hline
\end{tabular}

Table 6

Free vibrations of two-layered isotropic shell: fundamental frequency $f$ in $H z$ for $m=n=$ 10. Thermo-mechanical coupling parameter $\Delta(\%)=\frac{f_{T M}-f}{f} \times 100$ is put in brackets

\begin{tabular}{lcccc}
\hline$R_{\alpha} / h$ & 5 & 10 & 50 & 100 \\
\hline LD4 & 15148 & 14740 & 13729 & 12004 \\
LD4(TM) & $15149(0.007)$ & $14743(0.020)$ & $13736(0.051)$ & $12017(0.108)$ \\
ED4 & 15334 & 15145 & 13793 & 12042 \\
ED4(TM) & $15335(0.006)$ & $15146(0.007)$ & $13800(0.051)$ & $12056(0.116)$ \\
FSDT & 15441 & 15469 & 14540 & 12538 \\
FSDT(TM) & $15441(0.000)$ & $15469(0.000)$ & $14556(0.110)$ & $12569(0.247)$ \\
CLT & 15576 & 15593 & 15607 & 15609 \\
CLT(TM) & $15576(0.000)$ & $15593(0.000)$ & $15607(0.000)$ & $15609(0.000)$ \\
\hline
\end{tabular}

are $R_{\alpha} / h=5,10,50$ and 100, which means total thickness of the shell $h=2,1,0.2$ and $0.1 \mathrm{~m}$, respectively. Two different layered configurations are investigated for this free vibration problem. The first is a one-layered isotropic shell in Al2024 with Young's modulus $E=73 \mathrm{GPa}$, Poisson's ratio $\nu=0.3$ and mass density $\rho=2800 \mathrm{Kg} / \mathrm{m}^{3}$. The thermal properties are the specific heat per unit mass $C_{v}=897 \mathrm{~J} / \mathrm{Kg} \mathrm{K}$ and the thermal expansion coefficient $\alpha=25 \times 10^{-6} 1 / \mathrm{K}$. The two-layered isotropic shell has two layers of thickness $h_{1}=h_{2}=h / 2$, the bottom layer is in Al2024 and the top layer is in Ti22. The mechanical properties of the Ti22 layer are $E=110 G P a, \nu=0.32$ and $\rho=4420 \mathrm{Kg} / \mathrm{m}^{3}$. Its thermal properties are $C_{v}=560 \mathrm{~J} / \mathrm{Kg} \mathrm{K}$ and $\alpha=8.6 \times 10^{-6} 1 / \mathrm{K}$. By imposing the wave numbers $m$ and $n$ in the $\alpha$ and $\beta$ direction, respectively, the considered two-dimensional theory gives a number of frequencies equals the number of degrees of freedom through the thickness direction. In Table 1 , the degrees of freedom, and consequently the number of frequencies for fixed wave numbers, are given for CLT, FSDT and refined ESL and LW theories in the case of one-layered and two-layered shells. By increasing the wave numbers, it is possible to investigate the higher order modes [12].

\subsection{Results for fundamental frequencies}

The fundamental frequency in $\mathrm{Hz}$ is given in Tables 2, 3 and 4 for imposed wave numbers $m=n=1, m=n=$ 10 and $m=n=50$, respectively. These tables consider the one-layered shell: there are no differences between 
Table 7

Free vibrations of two-layered isotropic shell: fundamental frequency $f$ in $H z$ for $m=n=$ 50. Thermo-mechanical coupling parameter $\Delta(\%)=\frac{f_{T M}-f}{f} \times 100$ is put in brackets

\begin{tabular}{lcccc}
\hline$R_{\alpha} / h$ & 5 & 10 & 50 & 100 \\
\hline LD4 & 76991 & 76838 & 73750 & 71943 \\
LD4(TM) & $76992(0.001)$ & $76839(0.001)$ & $73763(0.018)$ & $71958(0.021)$ \\
ED4 & 77047 & 77023 & 75767 & 73559 \\
ED4(TM) & $77047(0.000)$ & $77024(0.001)$ & $75775(0.011)$ & $73574(0.020)$ \\
FSDT & 77168 & 77196 & 77378 & 77065 \\
FSDT(TM) & $77168(0.000)$ & $77196(0.000)$ & $77378(0.000)$ & $77082(0.022)$ \\
CLT & 77877 & 77965 & 78036 & 78045 \\
CLT(TM) & $77877(0.000)$ & $77965(0.000)$ & $78036(0.000)$ & $78045(0.000)$ \\
\hline
\end{tabular}
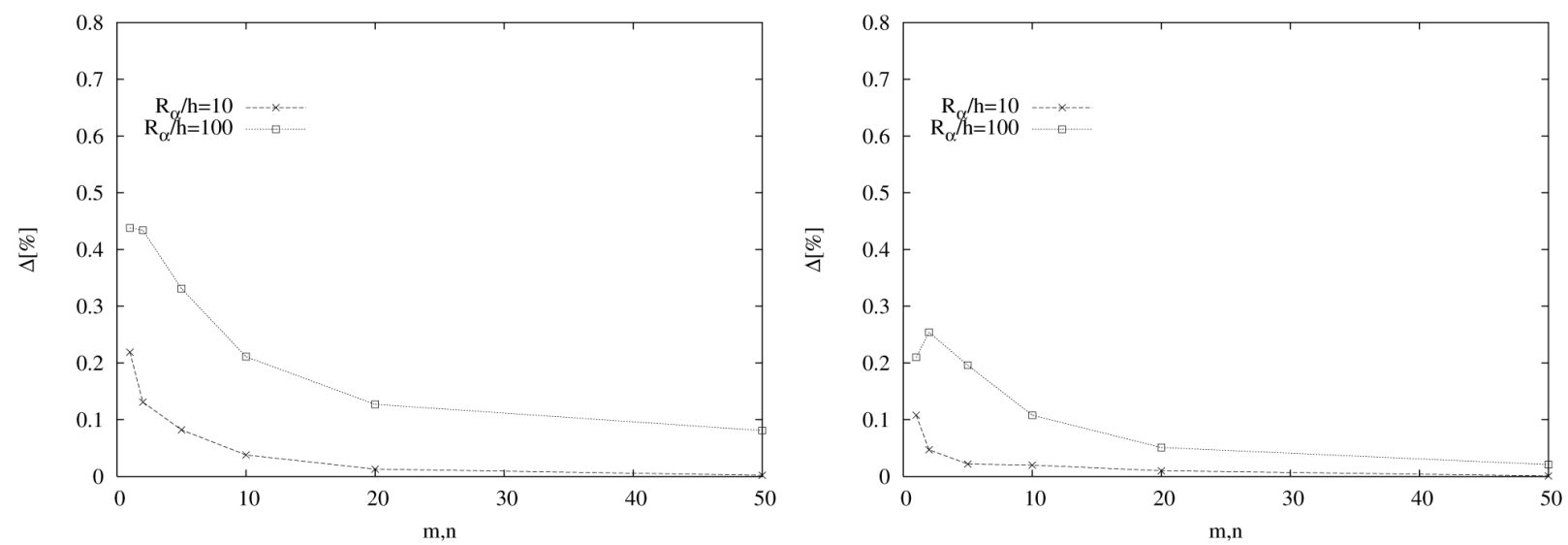

Fig. 3. Thermo-mechanical coupling in one-layered (on the left) and two-layered (on the right) isotropic shells for different values of wave number. Thermo-mechanical coupling parameter $\Delta(\%)=\frac{f_{T M}-f}{f} \times 100$ calculated for the fundamental frequency by using LD4 and LD4(TM) theories for $f$ and $f_{T M}$, respectively.
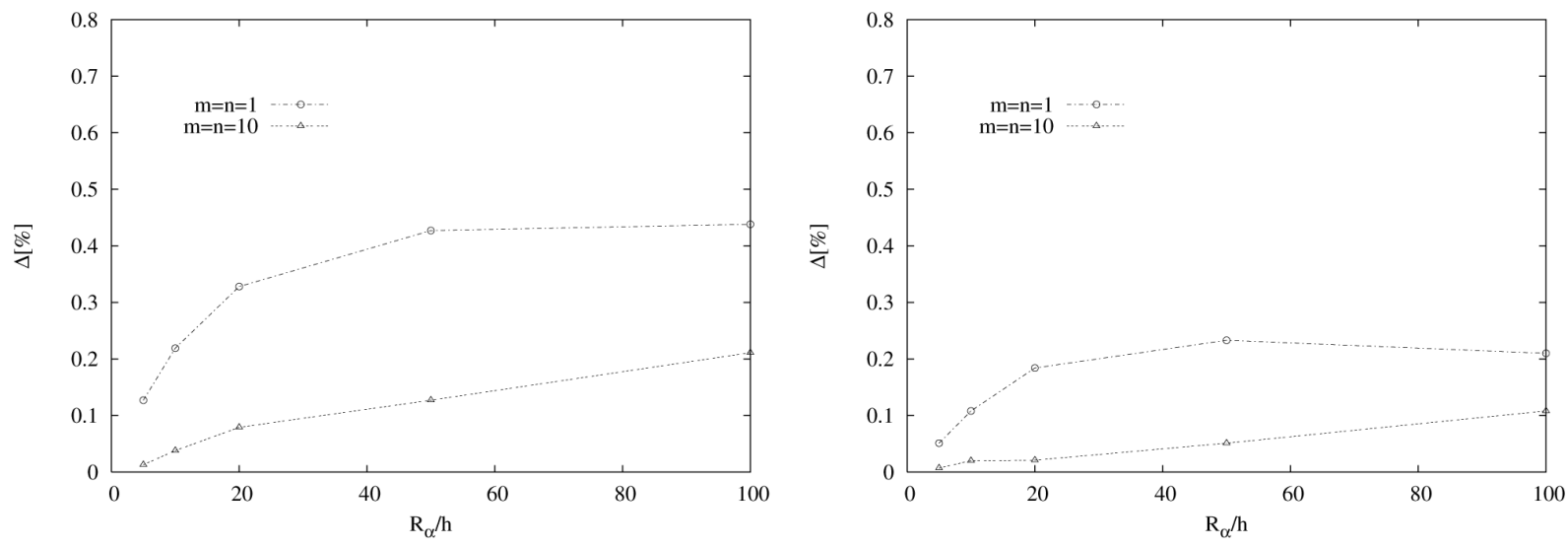

Fig. 4. Thermo-mechanical coupling in one-layered (on the left) and two-layered (on the right) isotropic shells for different values of thickness ratio. Thermo-mechanical coupling parameter $\Delta(\%)=\frac{f_{T M}-f}{f} \times 100$ calculated for the fundamental frequency by using LD4 and LD4(TM) theories for $f$ and $f_{T M}$, respectively.

the ESL and LW theories with the same order of expansion $N$ in the thickness direction. The comparison is made between the fundamental pure mechanical frequency $f$ and the fundamental thermo-mechanical frequency $f_{T M}$ for several thickness ratios $R_{\alpha} / h$ and for LD4, LD2, FSDT and CLT theories. The thermo-mechanical coupling, in terms of fundamental frequency, is evaluated by means of the parameter $\Delta(\%)=\frac{f_{T M}-f}{f} \times 100$. The parameter 
Table 8

Free vibrations of one-layered isotropic shell: higher-order frequency values $f$ in $H z$. Thickness ratio $R_{\alpha} / h=50$. Thermo-mechanical coupling parameter $\Delta_{i}(\%)=\frac{f_{T M i}-f_{i}}{f_{i}} \times 100$ is put in brackets

\begin{tabular}{|c|c|c|c|c|c|}
\hline & & & $m=1, n=1$ & & \\
\hline \multirow[t]{3}{*}{ LD4 } & 465.79 & 1590.5 & 2680.1 & 8077.5 & 8437.8 \\
\hline & 14360 & 15970 & 16607 & 29473 & 32906 \\
\hline & 33285 & 49163 & 49217 & 61542 & 91950 \\
\hline \multirow[t]{3}{*}{ LD4(TM) } & $467.78(0.427)$ & $1590.5(0.000)$ & $2692.3(0.455)$ & $8077.5(0.000)$ & $8440.7(0.034)$ \\
\hline & $14489(0.898)$ & $15970(0.000)$ & $16662(0.331)$ & 29794(1.089) & $32906(0.000)$ \\
\hline & $33325(0.120)$ & 49163(0.000) & $49222(0.010)$ & $62284(1.206)$ & $93061(1.208)$ \\
\hline FSDT & 468.38 & 1590.5 & 2688.6 & 8873.7 & 9260.0 \\
\hline FSDT(TM) & $472.42(0.862)$ & $1590.5(0.000)$ & $2714.2(0.952)$ & $8873.7(0.000)$ & $9267.2(0.078)$ \\
\hline CLT & 488.19 & 1590.5 & 2688.6 & & \\
\hline \multirow[t]{2}{*}{ CLT(TM) } & $492.78(0.940)$ & $1590.5(0.000)$ & $2714.2(0.952)$ & & \\
\hline & & & $m=10, n=10$ & & \\
\hline \multirow[t]{3}{*}{ LD4 } & 14124 & 15905 & 16187 & 17768 & 22483 \\
\hline & 22651 & 28124 & 33434 & 33595 & 36514 \\
\hline & 47947 & 51648 & 56396 & 66920 & 94165 \\
\hline \multirow[t]{3}{*}{ LD4(TM) } & $14142(0.127)$ & $15905(0.000)$ & $16207(0.124)$ & $17768(0.000)$ & $22483(0.000)$ \\
\hline & $22651(0.000)$ & $28275(0.537)$ & $33763(0.984)$ & $33887(0.869)$ & $36514(0.000)$ \\
\hline & $48244(0.619)$ & $51648(0.000)$ & $56754(0.635)$ & $67588(0.998)$ & $95332(1.239)$ \\
\hline FSDT & 14822 & 15905 & 18143 & 26885 & 28849 \\
\hline FSDT(TM) & $14847(0.169)$ & $15905(0.000)$ & $18143(0.000)$ & $27141(0.952)$ & $29075(0.783)$ \\
\hline CLT & 15905 & 23568 & 26885 & & \\
\hline CLT(TM) & $15905(0.000)$ & 23793(0.955) & $\begin{array}{c}27141(0.952) \\
m=10000, n=10000\end{array}$ & & \\
\hline \multirow[t]{3}{*}{ LD4 } & 15903850 & 15903880 & 15904380 & 15904420 & 15905140 \\
\hline & 15905160 & 15905930 & 15905950 & 15906460 & 15906500 \\
\hline & 29753380 & 29754350 & 29755770 & 29757240 & 29758260 \\
\hline \multirow[t]{3}{*}{ LD4(TM) } & $15903850(0.000)$ & $15903880(0.000)$ & $15904380(0.000)$ & $15904420(0.000)$ & $15905140(0.000)$ \\
\hline & $15905160(0.000)$ & $15905930(0.000)$ & $15905950(0.000)$ & $15906460(0.000)$ & $15906510(0.000)$ \\
\hline & $30112760(1.208)$ & $30113730(1.208)$ & $30115180(1.208)$ & $30116660(1.208)$ & $30117690(1.208)$ \\
\hline FSDT & 15904300 & 15905120 & 15905960 & 26883170 & 26885970 \\
\hline FSDT(TM) & $15904300(0.000)$ & $15905120(0.000)$ & $15905960(0.000)$ & $27139460(0.953)$ & $27142300(0.953)$ \\
\hline CLT & 15905110 & 16142950 & 26883170 & & \\
\hline CLT(TM) & $15905110(0.000)$ & $16142950(0.000)$ & $27139460(0.953)$ & & \\
\hline
\end{tabular}

$\Delta(\%)$ is always bigger than zero because the thermo-mechanical frequency is always larger than the pure mechanical frequency: the inclusion of the thermal part gives a bigger stiffness matrix. In Table 2 for lower order modes $m=n=1$, the maximum value of the thermo-mechanical coupling is about $0.4 \div 0.5 \%$ for thin shells, this value decreases for thick shells. LD2 and LD2(TM) theories have some difficulties to calculate the correct values of $f$ and $f_{T M}$, respectively, in particular for thick shells. However they give a satisfactory analysis of the coupling in terms of the parameter $\Delta(\%)$. Classical theories, such as FSDT and CLT, give a larger error for the evaluation of the pure mechanical and thermo-mechanical frequencies, in particular the evaluation of the thermo-mechanical frequency is not correct because such theories are not suitable to evaluate the thermal part. Moreover, the values of thermo-mechanical coupling parameter $\Delta(\%)$ given by FSDT and CLT are completely wrong. When the wave numbers increase, as in Tables 3 and 4 , the thermo-mechanical coupling decreases: it is about $0.2 \div 0.3 \%$ for the $m=n=10$ case (see Table 3 ) and less than $0.1 \%$ for the $m=n=50$ case (see Table 4 ). The dependency of the thermo-mechanical coupling by the thickness ratio is also confirmed for the higher values of wave numbers $(m, n)$. For higher order modes ( $m=n=10$ and $m=n=50$ ), the use of the LD4 theory is mandatory: the LD2 theory, in fact, exhibits some difficulties and CLT and FSDT are totally inadequate. The two-layered shell is considered in Tables 5, 6 and 7 for wave numbers $m=n=1, m=n=10$ and $m=n=50$, respectively. The maximum value of the parameter $\Delta(\%)$ is now smaller than the cases proposed for the one-layered shell: the embedded layer in $T i 22$ has smaller values of the specific heat per unit mass $C_{v}$ and thermal expansion coefficient $\alpha$ than those of the Al2024 layer, this means smaller thermo-mechanical coupling. In Table 5, for $m=n=1$, the maximum value of $\Delta(\%)$ is about $0.2 \%$, it decreases for $m=n=10$ and it is about $0.1 \%$ in Table 6 , in Table 7 (for $m=n=50$ ) the thermo-mechanical coupling is about $0.02 \div 0.03 \%$. All the considerations already made for the one-layered shells are confirmed for the two-layered case: the thermo-mechanical coupling depends on the imposed wave numbers and 
Table 9

Free vibrations of two-layered isotropic shell: higher-order frequency values $f$ in $H z$. Thickness ratio $R_{\alpha} / h=50$. Thermo-mechanical coupling parameter $\Delta_{i}(\%)=\frac{f_{T M i}-f_{i}}{f_{i}} \times 100$ is put in brackets

\begin{tabular}{|c|c|c|c|c|c|}
\hline & & & $m=1, n=1$ & & \\
\hline \multirow[t]{6}{*}{ LD4 } & 454.22 & 1560.7 & 2652.6 & 7987.0 & 8336.0 \\
\hline & 14138 & 15621 & 16326 & 23524 & 23575 \\
\hline & 29906 & 31231 & 31263 & 41516 & 41519 \\
\hline & 44768 & 59768 & 64515 & 64526 & 78849 \\
\hline & 81150 & 81205 & 96542 & 96574 & 123553 \\
\hline & 154142 & 184702 & & & \\
\hline \multirow[t]{7}{*}{ LD4(TM) } & $455.28(0.233)$ & $1560.7(0.000)$ & $2658.7(0.230)$ & $7987.0(0.000)$ & $8338.2(0.026)$ \\
\hline & $14399(1.846)$ & $15621(0.000)$ & $16371(0.276)$ & $23526(0.008)$ & $23575(0.000)$ \\
\hline & $30087(0.603)$ & $31231(0.000)$ & $31268(0.016)$ & 41518(0.005) & $41519(0.000)$ \\
\hline & $45135(0.820)$ & $60138(0.619)$ & $64518(0.005)$ & $64526(0.000)$ & 79455(0.769) \\
\hline & $81150(0.000)$ & $81213(0.010)$ & $96542(0.000)$ & $96575(0.001)$ & $124152(0.485)$ \\
\hline & $155423(0.831)$ & $185416(0.387)$ & & & \\
\hline & & & $\mathrm{m}=10, \mathrm{n}=10$ & & \\
\hline \multirow[t]{6}{*}{ LD4 } & 13729 & 15585 & 16119 & 17579 & 22021 \\
\hline & 22030 & 26775 & 28291 & 30339 & 31638 \\
\hline & 34880 & 37642 & 39916 & 44370 & 46762 \\
\hline & 52027 & 64065 & 66368 & 66894 & 82641 \\
\hline & 85097 & 87906 & 97787 & 101148 & 125951 \\
\hline & 155731 & 185678 & & & \\
\hline \multirow[t]{7}{*}{ LD4(TM) } & $13736(0.051)$ & $15585(0.000)$ & $16136(0.105)$ & $17579(0.000)$ & $22021(0.000)$ \\
\hline & $22030(0.000)$ & $26808(0.123)$ & $28291(0.000)$ & $30424(0.280)$ & $31784(0.461)$ \\
\hline & $34880(0.000)$ & $37747(0.279)$ & $40133(0.544)$ & $44370(0.000)$ & $46855(0.199)$ \\
\hline & $52360(0.640)$ & $64350(0.445)$ & $66368(0.000)$ & $67147(0.378)$ & $82641(0.000)$ \\
\hline & $85384(0.337)$ & $88267(0.411)$ & $97787(0.000)$ & $101217(0.068)$ & $126567(0.489)$ \\
\hline & $157030(0.834)$ & $186401(0.389)$ & & & \\
\hline & & & $\mathrm{m}=10000, \mathrm{n}=10000$ & & \\
\hline \multirow[t]{6}{*}{ LD4 } & 15420170 & 15420180 & 15420480 & 15420600 & 15420810 \\
\hline & 15420920 & 15421190 & 15421270 & 15610720 & 15611060 \\
\hline & 15905460 & 15905500 & 15905870 & 15905890 & 15906300 \\
\hline & 15906310 & 15906620 & 15906880 & 29756360 & 29757130 \\
\hline & 29757930 & 29758480 & 29889670 & 29971450 & 29971930 \\
\hline & 29972630 & 29973380 & & & \\
\hline \multirow[t]{6}{*}{ LD4(TM) } & $15420170(0.000)$ & $15420180(0.000)$ & $15420480(0.000)$ & $15420600(0.000)$ & $15420810(0.000)$ \\
\hline & $15420920(0.000)$ & $15421190(0.000)$ & $15421270(0.000)$ & $15610720(0.000)$ & $15611060(0.000)$ \\
\hline & $15905460(0.000)$ & $15905500(0.000)$ & $15905870(0.000)$ & $15905890(0.000)$ & $15906300(0.000)$ \\
\hline & $15906310(0.000)$ & $15906620(0.000)$ & $15906890(0.000)$ & $30047880(0.980)$ & $30048360(0.979)$ \\
\hline & $30049060(0.978)$ & $30049820(0.979)$ & $30063650(0.582)$ & $30115770(0.481)$ & $30116550(0.482)$ \\
\hline & $30117360(0.483)$ & $30117920(0.482)$ & & & \\
\hline
\end{tabular}

it increases with the thickness ratio. The shell in Tables 5, 6 and 7 is two-layered, therefore there are some differences between the ESL and LW theories with the same order of expansion in the thickness direction. ED4 theory works quite well for the $m=n=1$ case (in particular for thin shells with $R_{\alpha} / h=50,100$ ), but it exhibits some difficulties for higher order modes ( $m=n=10$ and $m=n=50)$. CLT and FSDT are totally inadequate for each thickness ratio $R_{\alpha} / h$ and for lower and higher values of the wave number. All the considerations made for Tables $2-7$, are confirmed and summarized in Figs 3 and 4. Figure 3 gives the variation in the thermo-mechanical coupling with the wave numbers for thick $\left(R_{\alpha} / h=10\right)$ and thin $\left(R_{\alpha} / h=100\right)$ one-layered and two-layered shells. Figure 4 discusses the variation in the thermo-mechanical coupling with the thickness ratio for wave numbers $m=n=1$ and $m=n=10$, in the case of one-layered and two-layered shells. The main conclusions are: $\Delta(\%)$ decreases with $(m, n)$ and increases with $R_{\alpha} / h, \Delta(\%)$ is larger for the one-layered shell.

\subsection{Results for higher order frequencies}

The results proposed in Tables 2-7, consider the fundamental frequencies $f$ and $f_{T M}$. In [3] a partial investigation has been made for the fundamental frequency in the case of wave numbers $m=n=1$. Now this study is extended to higher values of wave number and to further frequencies different from the fundamental one. The evaluation of 

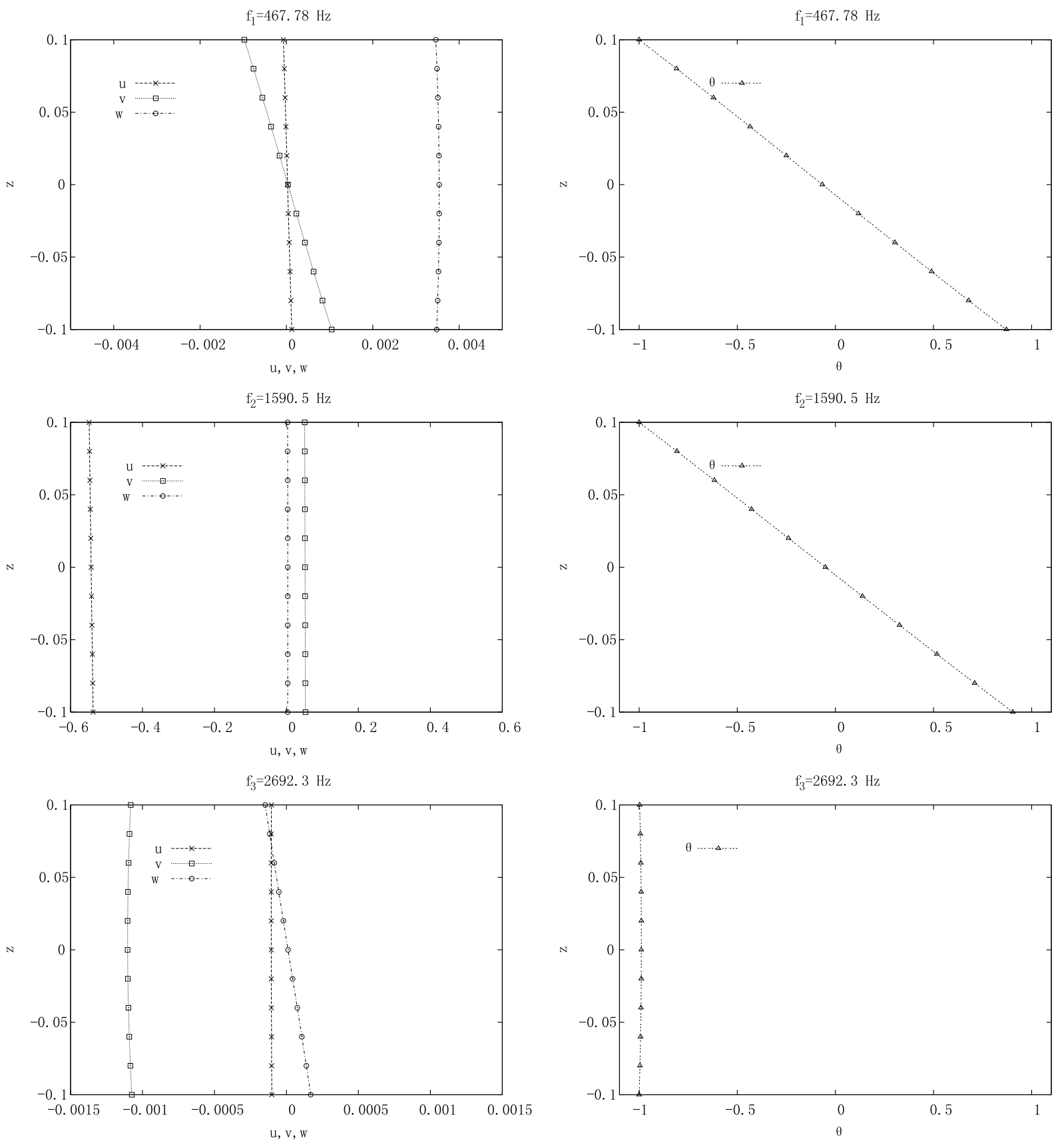

Fig. 5. One-layered isotropic shell, $m=n=1$ and $R_{\alpha} / h=50$. First three modes in terms of displacements and sovra-temperature. LD4(TM) model.

the thermo-mechanical coupling introduces different effects when all the vibration modes for fixed values of wave number are considered. Tables 8 and 9 give all the frequencies related to the degrees of freedom of the employed two-dimensional theory (see Table 1 ) for three different values of the wave numbers $(m=n=1, m=n=10$ and $m=n=10000$ ). Table 8 is for the one-layered shell: CLT gives three frequencies, FSDT gives five frequencies and LD4 gives 15 frequencies. Table 9 is for the two-layered plate: only the LD4 theory has been investigated and it gives 27 frequencies for each pair of $m$ and $n$. From Tables 8 and 9, it is clear that: when all the frequencies are considered (not only the fundamental one), the thermo-mechanical coupling does not depend on the considered wave numbers 

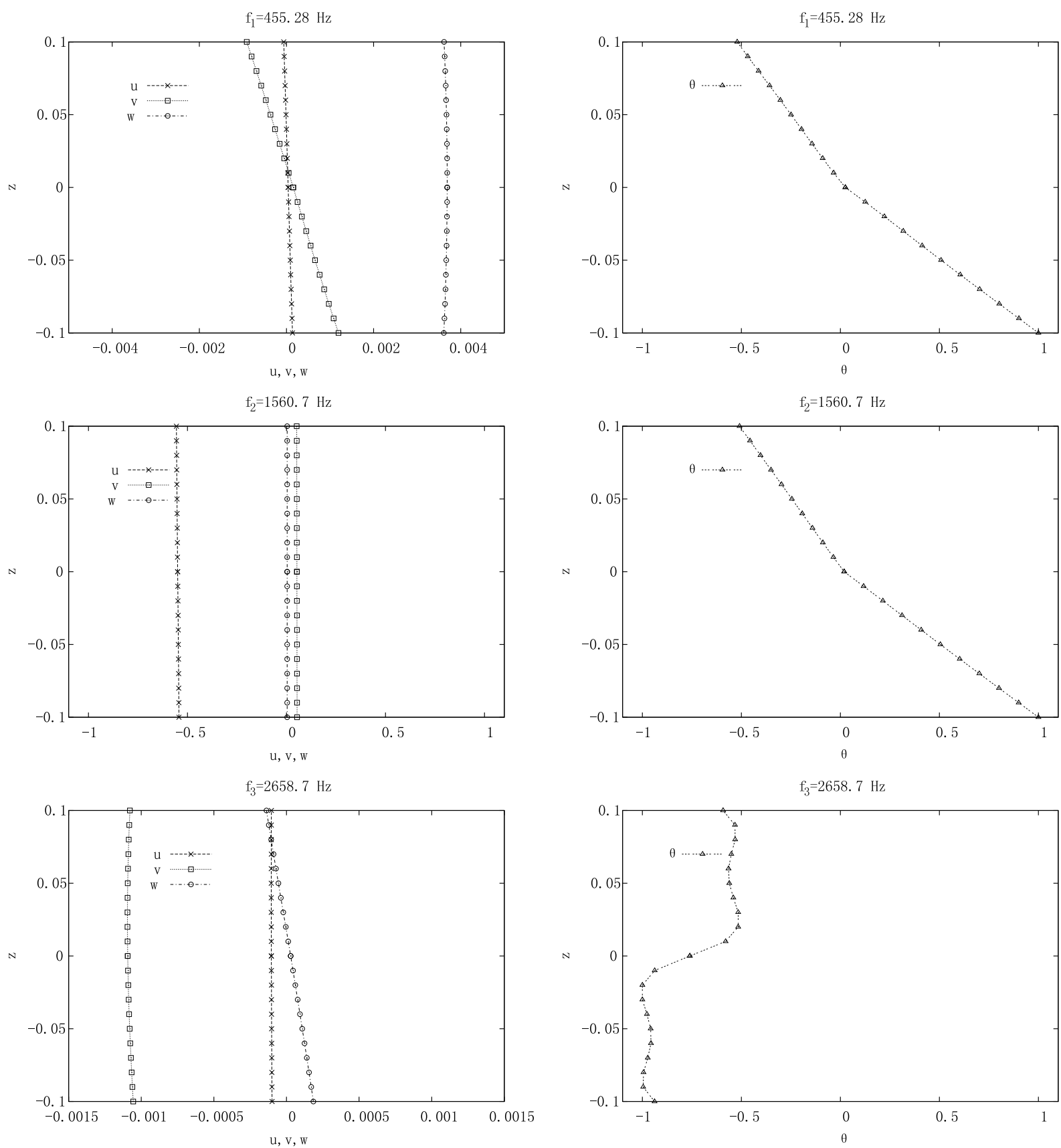

Fig. 6. Two-layered isotropic shell, $m=n=1$ and $R_{\alpha} / h=50$. First three modes in terms of displacements and sovra-temperature. LD4(TM) model.

$(m, n)$ and on the layers stacking, the coupling effect depends on the considered mode in terms of displacement and sovra-temperature. The use of refined theories, such as LD4, is mandatory for such an investigation. CLT and FSDT unfortunately lose some modes because of their reduced number of degrees of freedom. There are some frequencies which correspond to a particular vibration mode, where there is no thermo-mechanical coupling. This fact is clearly seen in Figs 5 and 6 for the case of one-layered and two-layered shells, respectively. Figures 5 and 6 give the first three vibration modes in terms of displacement components and sovra-temperature. The first three frequencies are given for thickness ratio $R_{\alpha} / h=50$ and $m=n=1$. For bending modes (related to $f_{1}$ and $f_{3}$ ), there is a 


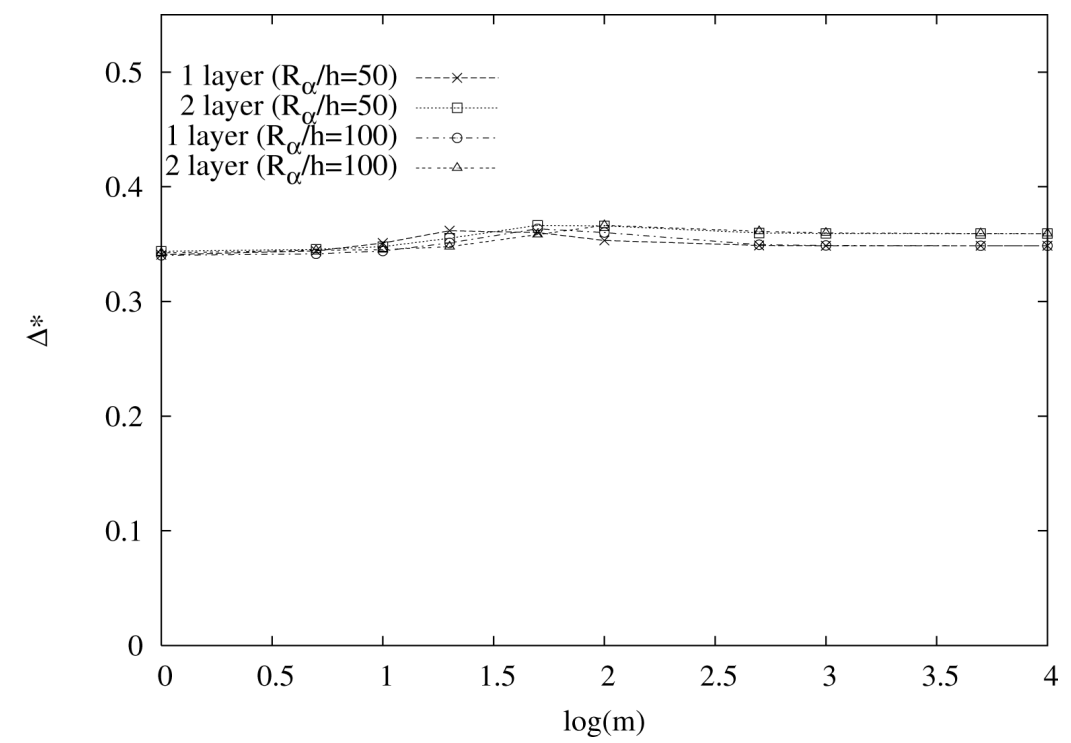

Fig. 7. Global energetic thermo-mechanical coupling $\Delta^{*}$ in one-layered and two-layered isotropic shells for different values of wave number

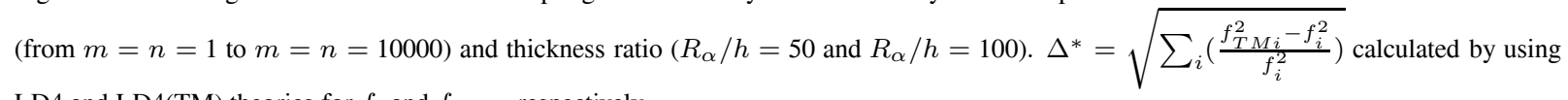
LD4 and LD4(TM) theories for $f_{i}$ and $f_{T M i}$, respectively.

thermo-mechanical coupling $\Delta(\%)$ (about $0.4 \%$ and $0.2 \%$, for the one-layered and two-layered shells, respectively). The second frequency is an in-plane mode: the mode in terms of transverse displacement $w$ is zero. The in-plane modes do not exhibit any thermo-mechanical coupling. For the plate case, investigated in [14], the mode in terms of transverse displacement $w$ equals zero means a mode in terms of sovra-temperature which is also zero. For the shell case, even when the mode in term of transverse displacement $w$ is zero, the mode in term of sovra-temperature is not zero because there is an effect given by the in-plane displacements $u$ and $v$ due to the curvature of the shell. However, for the in-plane vibrations the mode in terms of sovra-temperature does not give any thermo-mechanical coupling.

In the present work the new considerations about the vibration modes in Figs 5 and 6, and in Tables 8 and 9, create the necessity of defining a global energetic parameter to evaluate the thermo-mechanical coupling in such shells. A possible parameter is $\Delta^{*}=\sqrt{\sum_{i}\left(\frac{f_{T M i}^{2}-f_{i}^{2}}{f_{i}^{2}}\right)}$, which evaluates the thermo-mechanical coupling of all the obtained frequencies when the wave number is fixed and the theory is chosen. When a shell configuration is given (in particular its layers stacking), a two-dimensional theory is chosen and the wave numbers $(m, n)$ are imposed, the parameter $\Delta^{*}$ gives the global thermo-mechanical coupling. From Fig. 7, it is clear that the global thermo-mechanical coupling does not depend on the thickness ratio, on the layers stacking, and on the imposed wave numbers.

The results given in this work demonstrate how thermo-mechanical coupling is very small in the free vibration analysis of one-layered and two-layered metallic shells (the introduction of the curvature does not give further effects with respect to the plate case), and it can therefore be discarded: pure mechanical refined two-dimensional theories are sufficient to obtain a complete investigation of lower and higher order vibration modes for both thin and thick shells. However, the fully coupled thermo-mechanical models could be valid for thermography investigations: the increment in temperature is experimentally measured to determine the strains and stresses which have generated it [38-40].

\section{Conclusions}

The thermo-mechanical coupling in the free vibration analysis of one-layered and two-layered metallic shells has been investigated in this work. Such an investigation has been made by comparing the pure mechanical 
frequencies and coupled thermo-mechanical frequencies, these last have been obtained by using a fully coupled thermo-mechanical model where the temperature is also a primary variable in the governing equations and the thermal part is considered in the stiffness matrix. The introduction of the curvature does not give further effects with respect to the already investigated plate case. The main conclusions obtained in this work are listed as follows:

- the use of refined two-dimensional theories is mandatory to correctly determine the thermal and mechanical parts in the stiffness matrix for the thermo-mechanical investigations;

- the thermo-mechanical coupling is very small, less than the $0.5 \%$, and it can be discarded in free vibration analyses of shells. Such an analysis has never been performed in the open literature and it could play a fundamental role in thermography investigations;

- the thermo-mechanical coupling is zero for in-plane vibration modes, where the modes in terms of transverse displacement are zero;

- when the fundamental frequency is considered, the thermo-mechanical coupling has a limited dependence on the thickness ratio of the shell, its configuration (in particular the layer stacking), and the imposed wave numbers;

- if a global parameter for the thermo-mechanical coupling is opportunely defined, the thermo-mechanical coupling effect does not depend on the investigated case (layers stacking, thickness ratio and imposed wave numbers).

\section{References}

[1] J.L. Nowinski, Theory of Thermoelasticity with Applications, Sijthoff and Noordhoff, 1978, The Netherlands.

[2] S. Brischetto, E. Carrera, Coupled thermo-mechanical analysis of one-layered and multilayered plates, Composite Structures 92(8) (2010), 1793-1812.

[3] S. Brischetto and E. Carrera, Coupled thermo-mechanical analysis of one-layered and multilayered isotropic and composite shells, Computer Modeling in Engineering and Sciences CMES 56(3) (2010), 249-301.

[4] L. Librescu and P. Marzocca, Thermal stresses'03, vol.1, Virginia Polytechnic Institute and State University, 2003, Blacksburg, VA (USA).

[5] L. Librescu and Marzocca, Thermal stresses'03, vol.2, Virginia Polytechnic Institute and State University, 2003, Blacksburg, VA(USA).

[6] A.K. Noor and W.S. Burton, Computational models for high-temperature multilayered composite plates and shells, Applied Mechanics Reviews 45(10) (1992), 419-446.

[7] E. Carrera, Temperature profile influence on layered plates response considering classical and advanced theories, AIAA Journal 40(9) (2002), 1885-1896.

[8] E. Carrera, An assessment of mixed and classical theories for the thermal stress analysis of orthotropic multilayered plates, Journal of Thermal Stresses 23(9) (2000), 797-831.

[9] S. Brischetto, R. Leetsch, E. Carrera, T. Wallmersperger and B. Kröplin, Thermo-mechanical bending of functionally graded plates, Journal of Thermal Stresses 31(3) (2008), 286-308.

[10] S. Brischetto and E. Carrera, Thermal stress analysis by refined multilayered composite shell theories, Journal of Thermal Stresses 32(1) (2009), 165-186.

[11] S. Brischetto, Effect of the through-the-thickness temperature distribution on the response of layered and composite shells, International Journal of Applied Mechanics 1(4) (2009), 1-25.

[12] S. Brischetto and E. Carrera, Importance of higher order modes and refined theories in free vibration analysis of composite plates, Journal of Applied Mechanics 77(1) (2010), 1-14.

[13] E. Carrera and S. Brischetto, Vibrations of Plates and Shells in the Case of Thermo-Mechanical Coupling, The Seventh International Symposium on Vibrations of Continuous Systems, July 19-25, 2009, Zakopane, Poland.

[14] S. Brischetto and E. Carrera, Thermo-mechanical vibrations of one-layered and two-layered metallic plates, International Jounal of Applied Mechanics, in press, 2011.

[15] G.A. Altay and M.C. Dökmeci, Some variational principles for linear coupled thermoelasticity, International Journal of Solids and Structures 33(26) (1996), 3937-3948.

[16] G.A. Altay and M.C. Dökmeci, Fundamental variational equations of discontinuous thermopiezoelectric fields, International Journal of Engineering Sciences 34(7) (1996), 769-782.

[17] N.C. Das, S.N. Das and B. Das, Eigenvalue approach to thermoelasticity, Journal of Thermal Stresses 6(1) (1983), 35-43.

[18] A.A. Cannarozzi and F. Ubertini, A mixed variational method for linear coupled thermoelastic analyis, International Journal of Solids and Structures 38(4) (2001), 717-739.

[19] G.A. Altay and M.C. Dökmeci, Coupled thermoelastic shell equations with second sound for high-frequency vibrations of temperaturedependent materials, International Journal of Solids and Structures 38(16) (2001), 2737-2768.

[20] D. Givoli and O. Rand, Dynamic thermoelastic coupling effects in a rod, AIAA Journal 33(4) (1994), 776-778.

[21] E.V. Wilms and H. Cohen, Some one-dimensional problems in coupled thermoelasticity, Mechanics Research Communication 12(1) (1985), 41-47.

[22] J. Wauer, On magneto-thermo-elastic vibrations, Journal of Thermal Stresses 19(7) (1996), 671-691.

[23] W. Kosinski and K. Frischmuth, Thermomechanical coupled waves in a nonlinear medium, Wave Motion 34(1) (2001), 131-141. 
[24] D. Trajkoviski and R. Cukic, A coupled problem of thermoelastic vibrations of a circular plate with exact boundary conditions, Mechanics Research Communications 26(2) (1999), 217-224.

[25] Y.-L. Yeh, The effect of thermo-mechanical coupling for a simply supported orthotropic rectangular plate on non-linear dynamics, Thin-Walled Structures 43(8) (2005), 1277-1295.

[26] E. Carrera, A class of two-dimensional theories for multilayered plates analysis, Accademia delle Scienze di Torino, Memorie Scienze Fisiche 19-20, 1-39, 1995.

[27] E. Carrera, Theories and finite elements for multilayered plates and shells: a unified compact formulation with numerical assessments and benchmarking, Archivies of Computational Methods in Engineering 10(3) (2003), 215-296.

[28] J.N. Reddy, Mechanics of Laminated Composite Plates and Shells. Theory and Analysis, CRC Press, 2004, New York (USA).

[29] R.D. Mindlin, Influence of rotatory inertia and shear in flexural motions of isotropic elastic plates, Journal of Applied Mechanics 18 (1951), $31-38$.

[30] A.L. Cauchy, Sur l'équilibre et le mouvement d'une plaque solide, Exercise des Mathématique 3 (1828), 381-412.

[31] S.D. Poisson, Mémoire sur l'équilibre et le mouvement des corps élastique, Mémoires de l'Académie des Sciences des Paris 8 (1829), $357-570$.

[32] G. Kirchhoff, $\ddot{U}$ ber das gleichgewicht und die bewegung einer elastishen scheibe, Journal fur die reine und Angewandte Math 40 (1850), 51-88.

[33] E. Carrera and S. Brischetto, Analysis of thickness locking in classical, refined and mixed multilayered plate theories, Composite Structures 82(4) (2008), 549-562.

[34] E. Carrera and S. Brischetto, Analysis of thickness locking in classical, refined and mixed theories for layered shells, Composite Structures 85(1) (2008), 83-90.

[35] E. Carrera, S. Brischetto and P. Nali, Variational statements and computational models for multifield problems and multilayered structures, Mechanics of Advanced Materials and Structures 15(3) (2008), 182-198.

[36] A.W. Leissa, Vibration of Shells, NASA SP-288, 1973, Washington, D.C. (USA).

[37] T. Ikeda, Fundamentals of Piezoelectricity, Oxford University Press, 1990, Oxford (UK).

[38] C. Spiessberger, A. Gleiter and G. Busse, Aerospace Applications of Lockin-Thermography with Optical, Ultrasonic and Inductive Excitation, International Symposium on NDT in Aerospace, December 3-5, 2008, Fürth, Germany.

[39] G. Fantoni, L.G. Merletti and A. Salerno, Stato dell' arte della termografia Lock-In applicata a componenti di elicottero: analisi termoelastica e rilevazione di difetti, Il Giornale delle Prove non Distruttive, Monitoraggio, Diagnostica 4 (2008), 30-34.

[40] C. Ibarra-Castanedo, E. Grinzato, S. Marinetti, P. Bison, N. Avdelidis, M. Grenier, J.-M. Piau, A. Bendala and X. Maldague, Quantitative Assessment of Aerospace Materials by Active Thermography Techniques, 9th International Conference on Quantitative InfraRed Thermography, July 2-5, 2008, Krakow, Poland. 

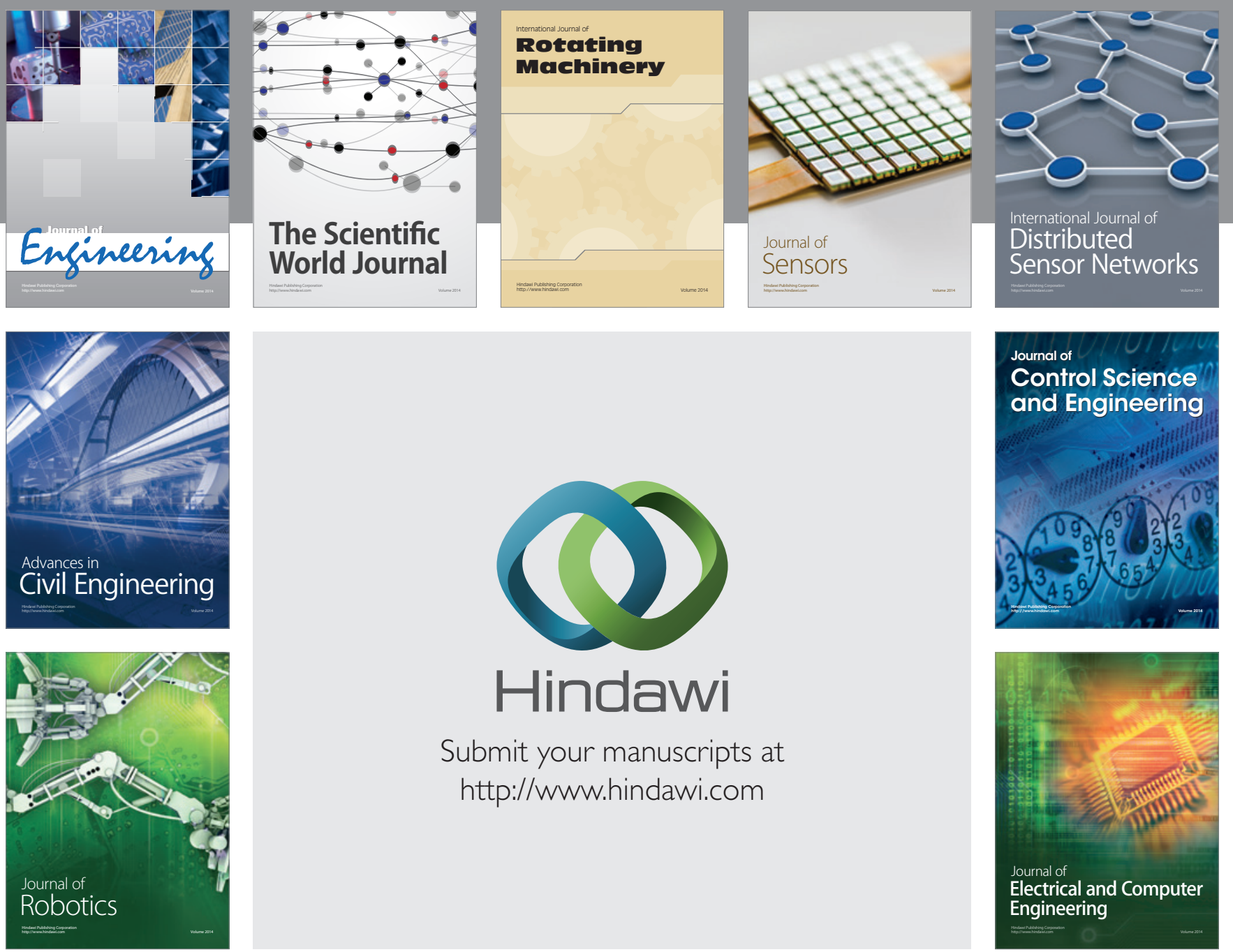

Submit your manuscripts at

http://www.hindawi.com
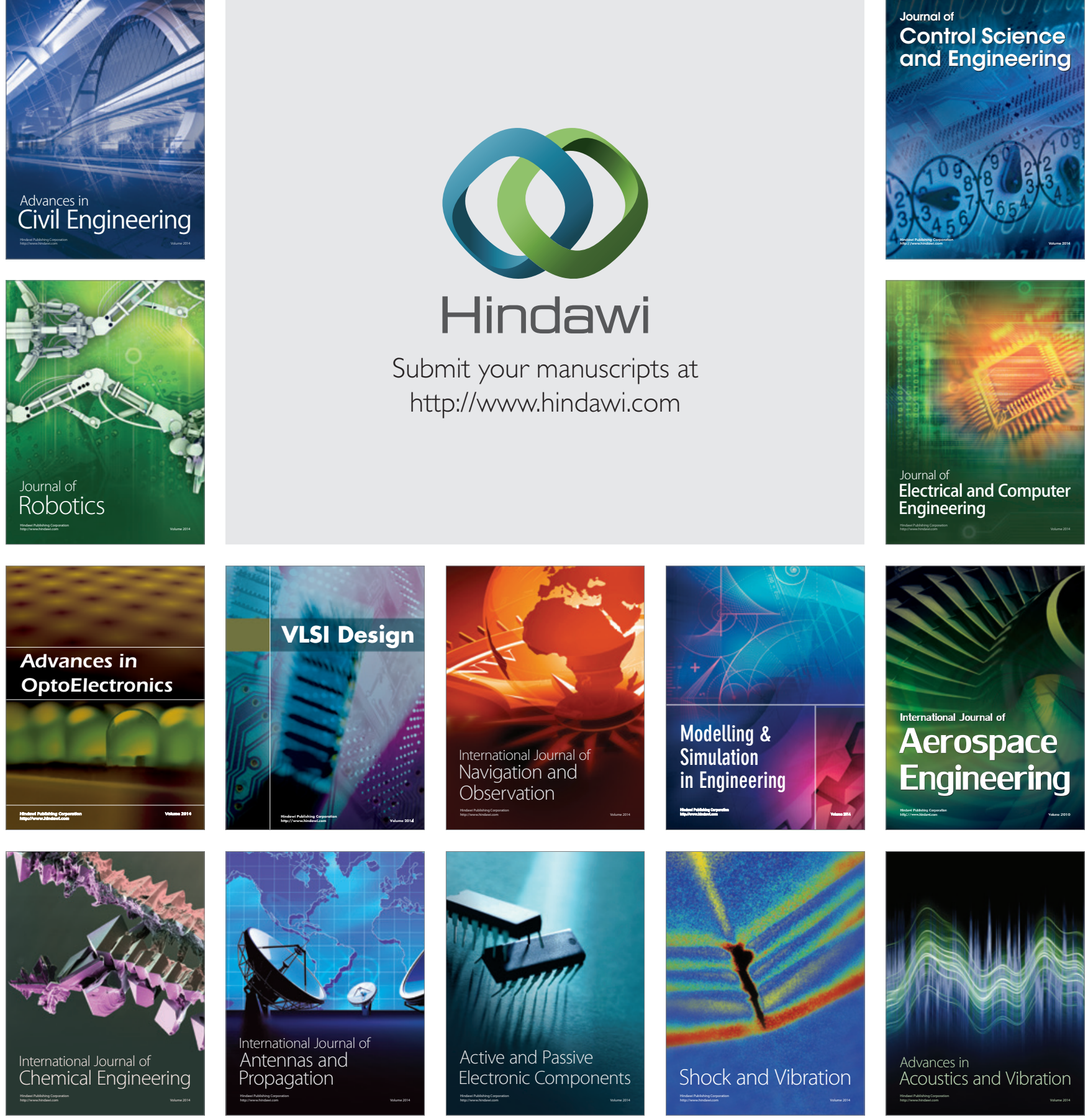